\title{
EXTREMAL LENGTH AND THE BOUNDARY BEHAVIOR OF CONFORMAL MAPPINGS
}

\author{
BURTON RODIN ${ }^{1}$ and S. E. WARSCHAWSKI ${ }^{2}$
}

\section{Introduction}

The Riemann mapping function $f: R \rightarrow S$ of a simply connected region $R$ onto a standard region $S$ sends a prime end $\zeta$ of $R$ to a boundary point of $S$. The classical boundary behavior problem is to find a more quantitative description by providing asymptotic estimates of $f(w)$ as $w \rightarrow \zeta$. The estimates will involve the geometry of $R$ near $\zeta$.

The problem originated with the question of extending the conformality of the mapping $f$ to a boundary point $\zeta$. The early results dealt with the behavior of $f$ at a corner $\zeta$, and with geometric conditions in a neighborhood of $\zeta$ which ensure the existence of the derivative of $f$, both for unrestricted approach to $\zeta$ as well as approach in a Stoltz angle ("angular derivative"). The study eventually evolved into the consideration of asymptotic estimates for $f$ under more general boundary configurations.

The present paper is a contribution to this study. It provides a number of basic estimates, under quite general conditions, which yield new or improved results when applied to special cases. Another aspect of the present paper is the systematic use of extremal length techniques. It is known that such techniques provide an efficient and powerful method of proof in this subject [2], [11], [13], [14]; it will be seen that they also provide a useful framework in which necessary and sufficient conditions can be given for various analytic properties of $f$.

The convenient normalization of Ahlfors [1] will be used: $S$ is a horizontal parallel strip and $f(\zeta)=+\infty$. Chapter I treats the problem in the abstract. This generality is needed because of the variety of special cases

\footnotetext{
1 Research partially supported by the National Science Foundation Grant No. GP38600.

2 Research partially supported by the National Science Foundation Grant No. GP32156.
} 
considered in subsequent chapters. The basic viewpoint in Chapter I is to assume that the approach $w \rightarrow \zeta$ is described in terms of a given set of cross cuts $\left\{V_{s}\right\}$ of $R$. If the extremal distances between the cross cuts satisfy certain conditions then estimates of $f(w)$ follow (Theorems 1 and 2).

In Chapter II these theorems are applied to the case that $R$ is a strip domain in the $w=u+i v$ plane and the given cross cuts $\left\{V_{s}\right\}$ are the Ahlfors cross cuts $\left\{\vartheta_{u}\right\}$. A number of theorems are obtained; one is a condition for $\operatorname{Re} f(w)$ to behave like the Ahlfors integral $\int u d u / \vartheta(u)$ (Theorem $3)$, and another is a condition for $\operatorname{Im} f(w)$ to be approximately linear on $\vartheta_{u}$ (Theorem 5). The former result leads to a short proof of a theorem of B. G. Eke [3] (see our Theorem 4). In $\$ 9$ the results from Chapter I are applied to the angular derivative problem. The result is Theorem 6; it provides necessary and sufficient conditions for the existence of an angular derivative. Although the conditions are not strictly geometric (they involve extremal length), the result seems to mark progress on this unsolved problem.

Chapter III is devoted to the proof of Theorem 8, an integral estimate for extremal distance in a quadrilateral. This estimate is used repeatedly in Chapter IV as a tool for applying the results of Chapter I to various types of smooth domains $R$. These applications lead to asymptotic estimates that are new or more general than previously known ones (Theorems $8,9,10)$.

\section{Contents}

I. General theorems $\ldots \ldots \ldots \ldots \ldots \ldots \ldots \ldots \ldots \ldots \ldots \ldots \ldots \ldots$

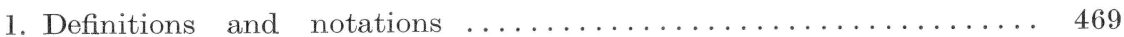

2. Lemmas ............................... 471

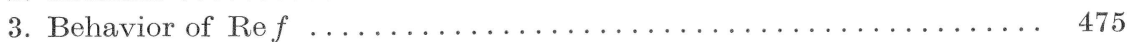

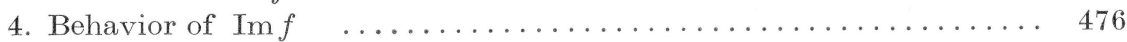

II. Applications to general strip domains $\ldots \ldots \ldots \ldots \ldots \ldots \ldots \ldots \ldots$

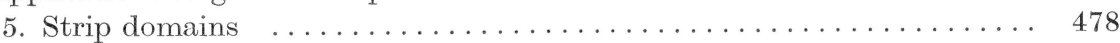

6. Boundedness of $E\left(u_{1}, u_{2}\right) \ldots \ldots \ldots \ldots \ldots \ldots \ldots \ldots \ldots \ldots \ldots \ldots$

7. Approximately linear behavior of $\operatorname{Im} f \ldots \ldots \ldots \ldots \ldots \ldots \ldots$

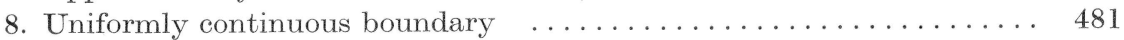

9. An application to the angular derivative problem $\ldots \ldots \ldots \ldots \ldots \ldots 4$

10. An angular derivative criterion $\ldots \ldots \ldots \ldots \ldots \ldots \ldots \ldots \ldots \ldots \ldots$

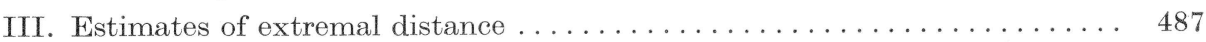

11. Definitions and notations $\ldots \ldots \ldots \ldots \ldots \ldots \ldots \ldots \ldots \ldots \ldots \ldots$

12. An extremal distance estimate $\ldots \ldots \ldots \ldots \ldots \ldots \ldots \ldots \ldots \ldots \ldots$

IV. Applications to domains with smooth boundaries . . . . . . . . . . 489

13. Strip domains bounded by smooth graphs $\ldots \ldots \ldots \ldots \ldots \ldots \ldots \ldots . \ldots 49$

14. Domains and surfaces generated by a smooth curve $\ldots \ldots \ldots \ldots . \ldots 492$

15. Symmetric strip domains $\ldots \ldots \ldots \ldots \ldots \ldots \ldots \ldots \ldots \ldots \ldots \ldots \ldots \ldots$

References ............................... 499 


\section{General theorems}

Section 1 describes the notations and basic assumptions for the abstract situation mentioned in the Introduction. It may be useful to mention here the specific cases covered by this abstraction. Riemann surfaces $R$, rather than plane regions, are needed only for $\S 14$. Cross cuts $\left\{V_{s}\right\}$ more general than the Ahlfors vertical cross cuts $\left\{\vartheta_{u}\right\}$ are needed only in $\S \S 14-15$. In the case that $R$ is a plane region the general cross cuts $\left\{V_{s}\right\}$ of this chapter could have been required to possess endpoints on $\partial R$, although no real simplifications result from doing so.

Sections 3 and 4 contain the main results of this chapter (Theorems 1 and 2).

1. Definitions and notations. Throughout this paper $R$ will denote a simply connected plane region or Riemann surface. $S$ will denote the parallel strip domain $\{z \mid-\infty<\operatorname{Re} z<+\infty, 0<\operatorname{Im} z<1\}$. $f$ is a given one-to-one conformal map of $R$ onto $S$. $\zeta$ is the boundary point or prime end of $R$ which corresponds under $f$ to $z=+\infty$ in $S$.

Let $\left\{V_{s} \mid s_{0} \leqq s<+\infty\right\}$ denote a family of disjoint cross cuts of $R$; "cross cut" is used here in the generalized sense that $V_{s}$ is an open Jordan arc in $R$ such that $R-V_{s}$ has two components. It is required that every sequence $\left\{V_{s_{n}}\right\}$, where $s_{n} \nearrow+\infty$, be a fundamental sequence of cross cuts for determining the prime end $\zeta$. In case $R$ is a plane region this requirement has the standard meaning from classical prime end theory. In case $R$ is a strip domain (Fig. 2; see Section 5), the Ahlfors cross cuts $\left\{\vartheta_{s}\right\}$ satisfy this requirement. It would not be relevant for the purposes of this paper to review the general theory of prime ends on simply connected Riemann surfaces in order to make the requirement more explicit; the consequences of it which we use in the remainder of this chapter are easily verified for each of the specific applications that are treated in Sections $5-10,13-15$. This viewpoint also applies to our use below of general notions of "ideal boundary."

Let $\left\{H_{t} \mid 0<t<1\right\}$ be a family of disjoint arcs on $R$ which have their initial points on $V_{s_{0}}$, and which tend to $\zeta$. Note that each $H_{t}$ intersects every $V_{s}$.

A quadrilateral $Q_{0}$ is a Jordan region with four distinguished boundary points called vertices. The vertices determine the four sides $\gamma_{i}(i=$ $1,2,3,4)$ of $Q_{0}$. We always assume that a definite ordering of the sides has been chosen. The ordering will be specified by the notation $Q_{0}=$ $Q\left(\gamma_{1}, \gamma_{2} ; \gamma_{3}, \gamma_{4}\right)$. Invariably when this notation is used $\gamma_{1}$ and $\gamma_{2}$ will be a pair of opposite sides of $Q_{0}$. Let $Q_{1}=Q\left(\beta_{1}, \beta_{2} ; \beta_{3}, \beta_{4}\right)$ be another quadrilateral. By a quadrilateral mapping of $Q_{0}$ into $Q_{1}$ we mean a $1-1$ 
conformal mapping of the interiors of $Q_{0}$ and $Q_{1}$ such that the continuous extension of the mapping to the closures sends $\gamma_{i}$ onto $\beta_{i}$ for $i=1,2,3,4$.

There is a self-explanatory way to indicate the various quadrilaterals determined by the arcs $\left\{V_{s}\right\}$ and $\left\{H_{t}\right\}$. For example, $Q\left(V_{s_{1}}, V_{s_{2}} ; H_{i_{1}}, H_{t_{2}}\right)$ is a quadrilateral whose four sides lie on $V_{s_{1}}, V_{s_{2}}, H_{t_{1}}$, and $H_{t_{2}}$. (If there is more than one such quadrilateral, additional specifications must be given so that it becomes uniquely determined. However, in all the special cases treated in the present paper each $V_{s}$ and $H_{t}$ intersect in exactly one point; this implies that the notation determines a unique quadrilateral. For simplicity, we now add this condition as a general requirement for $\left\{V_{s}\right\}$ and $\left\{H_{t}\right\}$. The quadrilateral notation therefore becomes unambiguous, and no further mention of uniqueness will be made.) This notation also specifies the ordering of the sides. We use a similar notation for quadrilaterals in the $x, y$-plane; for example,

$$
Q(x=a, x=b ; y=c, y=d)
$$

has the obvious interpretation.

We shall employ a self-explanatory notation for the extremal distance between a pair of opposite sides of a quadrilateral. For example, $\lambda\left(V_{s_{1}}, V_{s_{2}} ; H_{t_{1}}, H_{t_{2}}\right)$ denotes the extremal length in

$$
Q_{0}=Q\left(V_{s_{1}}, V_{s_{2}} ; H_{t_{1}}, H_{t_{2}}\right)
$$

of the family of arcs in $Q_{0}$ which join the sides of $Q_{0}$ that lie on $V_{s_{1}}$ and $V_{s_{2}}$.

It will be convenient to extend the notation for quadrilaterals to certain simply connected regions that need not be Jordan regions. Let $R_{0}$ be the component of $R-V_{s_{0}}$ which is a neighbourhood of $\zeta$. Its ideal boundary consists of $\zeta, V_{s_{0}}$, and two disjoint connected sets which we shall denote by $H_{0}$ and $H_{1}$. We choose the notation so that when $f$ is extended to the ideal boundary of $R_{0}$ it maps $H_{0}$ onto some ray $\left\{z \mid z>x_{0}\right\}$ and it maps $H_{1}$ onto a ray $\left\{z \mid \operatorname{Re} z>x_{0}^{\prime}, \operatorname{Im} z=1\right\}$. The symbol $Q\left(V_{s_{1}}, V_{s_{2}} ; H_{0}, H_{1}\right)$ denotes the simply connected subregion of $R$ whose ideal boundary consists of $V_{s_{1}}, V_{s_{2}}$, part of $H_{0}$, and part of $H_{1}$. The extremal length of all ares in this subregion which join $V_{s_{1}}$ to $V_{s_{2}}$ is denoted $\lambda\left(V_{s_{1}}, V_{s_{2}} ; H_{0}, H_{1}\right)$. The extremal length of all (open) ares in this subregion which separate the two sides lying on $V_{s_{1}}$ and $V_{s_{2}}$ is denoted by $\lambda\left(H_{0}, H_{1} ; V_{s_{0}}, V_{s_{1}}\right)$. Variations of the notation, for example $\lambda\left(H_{0}, H_{t} ; V_{s_{1}}, V_{s_{2}}\right)$ or $\lambda\left(V_{s_{1}}, V_{s_{2}} ; H_{t}, H_{1}\right)$, are self-explanatory. We may also speak of "quadrilateral mappings" of such subregions. 
Figures 1 and 2 may be useful for keeping track of the symbols introduced so far (in Figure 2, $\zeta$ is at $+\infty$ ).

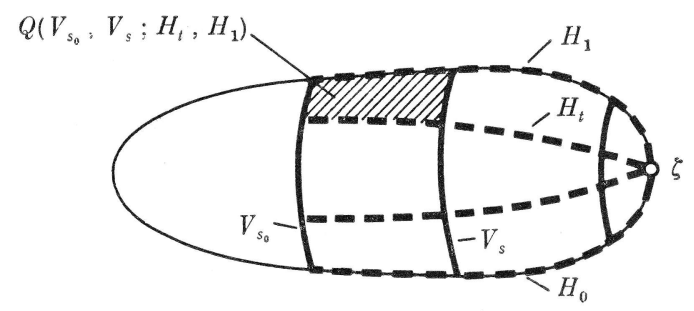

Figure 1

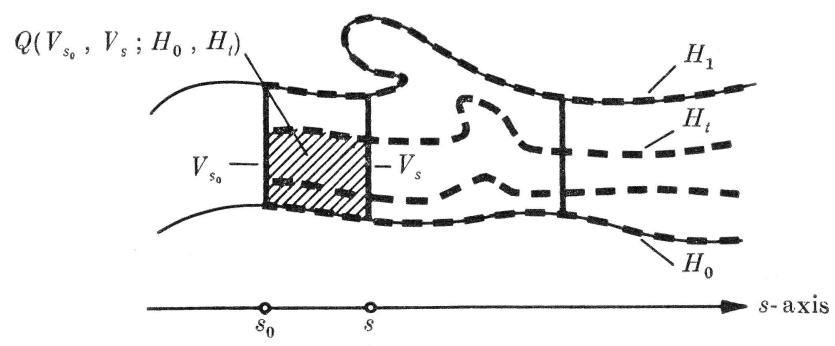

Figure 2

2. Lemmas. In this section we have collected together a number of lemmas for later use.

$\mathrm{L}$ e m m 1. Given $a>0$ and $\varepsilon>0$, there is $a \quad \delta_{1}=\delta_{1}(a, \varepsilon)>0$ with the following property. Let $A \geqq a$ and let $g$ be the quadrilateral mapping of $Q_{1}=Q(x=0, x=A ; y=0, y=1)$ onto $Q_{2}=Q\left(\gamma_{1}, \gamma_{2} ; y=0, y=1\right)$ where $\gamma_{1}$ and $\gamma_{2}$ are disjoint arcs which join $\{y=0\}$ to $\{y=1\}$. If

$$
\left|\operatorname{Re} z_{1}\right| \leqq \delta_{1}, \quad\left|A-\operatorname{Re} z_{2}\right| \leqq \delta_{1} \quad\left(\text { all } z_{1} \in \gamma_{1}, z_{2} \in \gamma_{2}\right)
$$

then $|g(z)-z|<\varepsilon$ for all $z \in Q_{1}$ with $|\operatorname{Re} z-(a / 2)|<a / 4$.

Proof. Let $G(z)=g(z)-z$. If $(2.1)$ holds then $\operatorname{Re} G$ is bounded in absolute value by $\delta_{1}$ on the vertical sides of $Q_{1}$. Since it has vanishing normal derivative on the horizontal sides, it satisfies the maximum principle in $Q_{1}$, and hence $|\operatorname{Re} G|<\delta_{1}$ in

$$
Q^{\prime}=Q(x=0, x=a ; y=0, y=1) \subset Q_{1} .
$$

Therefore, if the theorem were false for a particular $a>0$ and $\varepsilon>0$, we could obtain a sequence $\left\{G_{n}\right\}$ of analytic functions on $Q^{\prime}$ such that $\operatorname{Im} G_{n}=0$ on the horizontal sides of $Q^{\prime}, \operatorname{Re} G_{n} \rightarrow 0$ in $Q^{\prime}$, and $\left|G_{n}(z)\right| \geqq \varepsilon$ for some $z$ with $|\operatorname{Re} z-(a / 2)|<a / 4$; a contradiction. 
L e m m 2. Let $s^{\prime}>s_{0}$ be given. To each $\varepsilon>0$ there is an $a_{2}=$ $a_{2}(\varepsilon)>s^{\prime}$ with the following property. If $b>a>a_{2}$, then for all sufficiently large $s^{\prime \prime}$ one has

$$
|f(w)+C-\psi(w)|<\varepsilon
$$

for all $w \in Q\left(V_{a}, V_{b} ; H_{0}, H_{1}\right)$. Here $\psi$ is the quadrilateral mapping of $Q\left(V_{s^{\prime}}, V_{s^{\prime \prime}} ; H_{0}, H_{1}\right)$ onto the rectangle $Q(x=0, x=A ; y=0, y=1)$ where $A=\lambda\left(V_{s^{\prime}}, V_{s^{\prime \prime}} ; H_{0}, H_{1}\right) ; C$ is a real constant.

Proof. Let $\left\{s_{n}\right\}$ be an arbitrary sequence of real numbers which tend to $+\infty$. It suffices to prove that the lemma holds when $s^{\prime \prime}$ is replaced by $s_{n}$. Let $\psi_{n}(w)$ be the quadrilateral mapping $\psi$ when $s^{\prime \prime}$ is replaced by $s_{n}$. For any $s$, let $\tilde{R}_{s}$ denote that component of $R-V_{s}$ which contains $\zeta$ as a prime end.

(i) Suppose $s^{\prime}>s_{0}$ is given. There exists a one-to-one conformal map $F$ of $\tilde{R}_{s^{\prime}}$ onto the halfstrip

$$
S_{1}=\left\{z_{1}=x_{1}+i y_{1} \mid x_{1}>0,0<y_{1}<1\right\}
$$

such that the continuous extension of $F$ to $V_{s^{\prime}}$ sends $V_{s^{\prime}}$ onto $\left\{x_{1}=0,0<y<1\right\}$. $F$ maps $Q\left(V_{s^{\prime}}, V_{s_{n}} ; H_{o}, H_{1}\right)$ onto a quadrilateral $D_{n} \subset S_{1}$. Given $\alpha>0$, the rectangle $\left\{0 \leqq x_{1} \leqq \alpha, 0 \leqq y \leqq 1\right\}$ will be contained in the closure of $D_{n}$ if $n$ is sufficiently large.

Let $z_{2}=\varphi_{n}\left(z_{1}\right)$ be the quadrilateral mapping of $D_{n}$ onto the rectangle $P_{n}$ in the $z_{2}=x_{2}+i y_{2}$ plane, $P_{n}=Q\left(x_{2}=0, x_{2}=A_{n} ; y_{2}=0, y_{2}=1\right)$; here $A_{n}=\lambda\left(V_{s^{\prime}}, V_{s_{n}} ; H_{0}, H_{1}\right) \rightarrow+\infty$ as $n \rightarrow \infty$. By reflection across the lines $\left\{y_{1}=0\right\},\left\{y_{1}=1\right\}$, and then $\left\{x_{1}=0\right\}$, we can extend $\varphi_{n}$ to a one-to-one conformal map of a region $D_{n}^{*}$ onto the region $P_{n}^{*}=$ $\left\{-A_{n}<x_{2}<A_{n},-1<y_{2}<2\right\}$. The extended functions $\varphi_{n}$ all have the properties $\varphi_{n}(i)=i$ and $\varphi_{n}^{\prime}(i)>0$. Since $A_{n} \rightarrow+\infty$ it follows from the Carathéodory kernel theorem that $\lim _{n \rightarrow \infty} \varphi_{n}\left(z_{1}\right)=z_{1}$ uniformly on any compact subset of $\left\{-\infty<x_{1}<\infty,-1<y<2\right\}$.

Thus, given $\varepsilon>0$ and $\alpha>0$ there is an $N=N(\varepsilon, \alpha)$ such that

$$
\begin{aligned}
\left|\varphi_{n}\left(z_{1}\right)-z_{1}\right| & <\frac{\varepsilon}{2} \quad \text { if } \\
0 & \leqq \operatorname{Re} z_{1} \leqq \alpha, 0 \leqq \operatorname{Im} y_{1} \leqq 1, \text { and } n \geqq N(\varepsilon, \alpha) .
\end{aligned}
$$

(ii) The function $z=f(w)$ maps $\tilde{R}_{s^{\prime}}$ onto the component of $S-f\left(V_{s^{\prime}}\right)$ which contains $z=+\infty$. Let $z_{1}=h(z)$ map $f\left(\tilde{R}_{s^{\prime}}\right)$ one-toone and conformally onto the halfstrip $S_{1}=\left\{x_{1}>0,0<y_{1}<1\right\}$ so that its continuous extension carries $f\left(V_{s^{\prime}}\right)$ onto $\left\{x_{1}=0,0<y_{1}<1\right\}$ and $z=+\infty$ onto $z_{1}=+\infty$. Thus $F(w)=h(f(w))$. Since $h$ is dif- 
ferentiable at $z=+\infty$ there is a real constant $C$ such that $\lim _{x \rightarrow+\infty}[h(z)-z]=C$, uniformly in $\{0 \leqq y \leqq 1\}$. Thus, given $\varepsilon>0$ there is an $x(\varepsilon)$ such that

$$
|h(z)-z-C|<\frac{\varepsilon}{2} \quad \text { if } \operatorname{Re} z>x(\varepsilon), 0 \leqq \operatorname{Im} z \leqq 1 .
$$
Then

Now choose $a_{2}=a_{2}(\varepsilon)>s^{\prime}$ so that $\operatorname{Re} f(w)>x(\varepsilon)$ whenever $w \in R_{a_{2}}$.

$$
|h(f(w))-f(w)-C|<\frac{\varepsilon}{2} \quad \text { if } w \in R_{a_{2}} .
$$

(iii) Finally, let $a, b$ be given with $a_{2}(\varepsilon)<a<b$. Choose $\alpha>0$ so that the image of $Q\left(V_{a}, V_{b} ; H_{0}, H_{1}\right)$ under $F$ is contained in the rectangle $\left\{0<x_{1}<\alpha, 0<y_{1}<1\right\}$. If $n \geqq N(\varepsilon, \alpha)$ then by (2.3)

$$
\left|\varphi_{n}(F(w))-F(w)\right|<\frac{\varepsilon}{2} \quad\left(w \in Q\left(V_{a}, V_{b} ; H_{0}, H_{1}\right)\right) .
$$

or, since $\psi_{n}(w)=\varphi_{n}(F(w))$ and $F(w)=h(f(w))$,

$$
\left|\psi_{n}(w)-h(f(w))\right|<\frac{\varepsilon}{2} \quad\left(w \in Q\left(V_{a}, V_{b} ; H_{0}, H_{1}\right)\right) .
$$

From (2.4) and (2.5) we obtain the desired conclusion,

$$
\left|f(w)+C-\psi_{n}(w)\right|<\varepsilon .
$$

$\mathrm{L}$ e $\mathrm{m} \mathrm{m}$ a 3 . Given $\varepsilon>0$, there is a $\delta_{3}=\delta_{3}(\varepsilon)>0$ with the following property. Let $\gamma$ be an arc in a rectangle $Q=Q(x=0, x=A ; y=0, y=1)$ such that $\gamma$ separates the vertical sides of $Q$. Let

$\lambda^{\prime}=\lambda(x=0, \gamma ; y=0, y=1) \quad$ and $\quad \lambda^{\prime \prime}=\lambda(\gamma, x=A ; y=0, y=1)$. If $\lambda^{\prime}+\lambda^{\prime \prime} \geqq A-\delta_{3}$ then $\left|\operatorname{Re} z-\lambda^{\prime}\right| \leqq \varepsilon$ for all $z \in \gamma$.

Proof. Reflect $\gamma$ and $Q$ across the real axis to obtain $\bar{\gamma}$ and $\bar{Q}$. Map $Q \cup \bar{Q}$ into the annulus $\left\{1<|w|<e^{\pi A}\right\}$ by $w=e^{\pi z}$. The closure of $w(\gamma \cup \bar{\gamma})$ is a continuum which separates the contours of the annulus. In this way one can derive the lemma from Teichmüller's Modulsatz [17, p. 649].

L e m m 4. Let $M>0$ be fixed. To each $\varepsilon>0$ there is a $\delta_{4}=$ $\delta_{4}(\varepsilon)>0$ with the following property. If $A \leqq M$, and if $\gamma$ is an arc in $Q=Q(x=0, x=A ; y=0, y=1)$ which joins the two vertical sides of $Q$, and if

$$
\frac{\lambda(y=0, \gamma ; x=0, x=A)}{\lambda(y=0, y=1 ; x=0, x=A)} \geqq t-\delta_{4}
$$


and

$$
\frac{\lambda(\gamma, y=1 ; x=0, x=A)}{\lambda(y=0, y=1 ; x=0, x=A)} \geqq 1-t-\delta_{4},
$$

then $|\operatorname{Im} z-t| \leqq \varepsilon$ for all $z \in \gamma$.

Proof. We simplify the notation so that the left-hand terms in (2.6) and (2.7) become $\lambda^{\prime} / \lambda$ and $\lambda^{\prime \prime} / \lambda$. Thus $A=\lambda^{-1}$ and $M \lambda \geqq 1$. Without loss of generality we assume $M \geqq 2$.

Given $\varepsilon>0$, choose $\delta_{4} \leqq \delta_{3}(\varepsilon / 2 M) / 2$ where $\delta_{3}$ is determined by Lemma 3.

If $\lambda>1$ choose a positive integer $n$ such that $2^{n-1}<\lambda \leqq 2^{n}$. If $\lambda \leqq 1$ choose $n=0$. Reflect $Q$ across $\{\operatorname{Re} z=1 / \lambda\}$ to obtain $Q^{*}$, and set $Q_{1}=Q \cup Q^{*}$. Reflect $Q_{1}$ across $\{\operatorname{Re} z=2 / \lambda\}$ to obtain $Q_{1}^{*}$, and set $Q_{2}=Q_{1} \cup Q_{1}^{*}$. Continue in this way until

$$
\tilde{Q}=Q\left(x=0, x=2^{n} / \lambda ; y=0, y=1\right)
$$

is obtained. The images of $\gamma$ under these reflections piece together to form an arc $\tilde{\gamma}$ which joins the vertical sides of $\tilde{Q}$.

Map $\tilde{Q}$ onto $Q^{\prime}=Q\left(u=0, u=\lambda / 2^{n} ; v=0, v=1\right)$ by $w=\psi(z)=$ $i\left(1-2^{-n} z \lambda\right)$. Then $\psi(\tilde{\gamma})$ separates $Q^{\prime}$ into two quadrilaterals with extremal distances

$$
\begin{gathered}
\lambda(u=0, \psi(\tilde{\gamma}) ; v=0, v=1)=\frac{\lambda^{\prime}}{2^{n}}, \\
\lambda\left(\psi(\tilde{\gamma}), u=\frac{\lambda}{2^{n}} ; v=0, v=1\right)=\frac{\lambda^{\prime \prime}}{2^{n}} .
\end{gathered}
$$

The hypotheses (2.6) and (2.7) yield $\lambda^{\prime}+\lambda^{\prime \prime} \geqq \lambda-2 \lambda \delta_{4}$. Since $2^{-n} \lambda \leqq 1$ this implies

$$
\frac{\lambda^{\prime}}{2^{n}}+\frac{\lambda^{\prime \prime}}{2^{n}} \geqq \frac{\lambda}{2^{n}}-2 \delta_{4} \geqq \frac{\lambda}{2^{n}}-\delta_{3}\left(\frac{\varepsilon}{2 M}\right) .
$$

'Therefore, when we apply Lemma 3 to the two quadrilaterals in $Q^{\prime}$, we obtain $\left|\operatorname{Re} w-2^{-n} \lambda^{\prime}\right| \leqq \varepsilon / 2 M$ for all $w \in \psi(\tilde{\gamma})$. When this result is transferred back to $Q$ via $\psi^{-1}$ we obtain $\left|2^{-n} \lambda \operatorname{Im} z-2^{-n} \lambda^{\prime}\right| \leqq \varepsilon / 2 M$ for $z \in \tilde{\gamma}$, or

$$
\left|\operatorname{Im} z-\frac{\lambda^{\prime}}{\lambda}\right| \leqq \frac{2^{n} \varepsilon}{2 M \lambda} \leqq \frac{\varepsilon}{2} \quad(z \in \tilde{\gamma}) .
$$

The subadditivity of extremal distance, $\lambda^{\prime}+\lambda^{\prime \prime} \leqq \lambda$, together with (2.6) and (2.7), implies that $\left|\left(\lambda^{\prime} \mid \lambda\right)-t\right| \leqq \delta_{4}$. When combined with (2.9) this gives 


$$
|\operatorname{Im} z-t| \leqq \delta_{4}+\frac{\varepsilon}{2}
$$

for $z \in \tilde{\gamma}$ and, a fortiori, for $z \in \gamma$. We originally chose $\delta_{4} \leqq \delta_{3}(\varepsilon / 2 M) / 2$. We may require, in addition, that $\delta_{4} \leqq \varepsilon / 2$. Then the bound in (2.10) is $\leqq \varepsilon$ as desired.

3. Behavior of $\operatorname{Re} f$. In this section we give a condition on the cross cuts $\left\{V_{s}\right\}$ which leads to an asymptotic estimate for the real part of the strip mapping $f$.

Condition I. Given $\varepsilon>0$ there is an $s_{I}=s_{I}(\varepsilon)>s_{0}$ such that if $s_{2}>s>s_{1}>s_{I}$ then

$$
\lambda\left(V_{s_{1}}, V_{s} ; H_{0}, H_{1}\right)+\lambda\left(V_{s}, V_{s_{2}} ; H_{0}, H_{1}\right) \geqq \lambda\left(V_{s_{1}}, V_{s_{2}} ; H_{0}, H_{1}\right)-\varepsilon .
$$

$\mathrm{T} \mathrm{h}$ e o r e $\mathrm{m}$ 1. Assume Condition I holds. Then there is a real constant $C$ such that

$$
\lim _{\substack{s \rightarrow+\infty \\ w \in V_{s}}}\left[\operatorname{Re} f(w)-\lambda\left(V_{s_{0}}, V_{s} ; H_{0}, H_{1}\right)\right]=C .
$$

Proof. Let $\varepsilon>0$ be given. Determine $\delta_{3}=\delta_{3}(\varepsilon / 4)$ according to Lemma 3 ; determine $s_{I}=s_{I}\left(\delta_{3}\right)$ according to Condition I; determine $a_{2}=a_{2}(\varepsilon / 4)>s^{\prime}=s_{I}$ according to Lemma 2 . We shall first show that if $s>a_{2}$ then the horizontal oscillation of $f\left(V_{s}\right)$ is less than $\varepsilon$; the horizontal oscillation of a set $U$ is the quantity

$$
\sup \left\{\operatorname{Re} z_{1}-\operatorname{Re} z_{2} \mid z_{1} \in U, z_{2} \in U\right\} .
$$

Given $s>a_{2}$, choose $a$ and $b$ so that $a_{2}<a<s<b$. For sufficiently large $s^{\prime \prime}$,

$$
|f(w)+C-\psi(w)|<\frac{\varepsilon}{4} \quad\left(w \in Q\left(V_{a}, V_{b} ; H_{0}, H_{1}\right)\right)
$$

by Lemma 2, where $\psi$ is the quadrilateral mapping of $Q^{\prime}=$ $Q\left(V_{s^{\prime}}, V_{s^{\prime \prime}} ; H_{0}, H_{1}\right) \quad$ onto $Q^{\prime \prime}=Q(x=0, x=A ; y=0, y=1)$. Let $\lambda^{\prime}=\lambda\left(V_{s^{\prime}}, V_{s} ; H_{0}, H_{1}\right), \quad \lambda^{\prime \prime}=\lambda\left(V_{s}, V_{s^{\prime \prime}} ; H_{0}, H_{1}\right)$, and $\lambda=$ $\lambda\left(V_{s^{\prime}}, V_{s^{\prime \prime}} ; H_{0}, H_{1}\right)$. Since $a_{2}>s_{I}\left(\delta_{3}\right)$, Condition I implies that $\lambda^{\prime}+\lambda^{\prime \prime}$ $\geqq \lambda-\delta_{3}$. Since $\psi$ preserves extremal distances, a corresponding inequality holds for the components of $Q^{\prime \prime}-\psi\left(V_{s}\right)$. Lemma 3 therefore implies $\left|\operatorname{Re} \psi(w)-\lambda^{\prime}\right| \leqq \varepsilon / 4$ for all $w \in V_{s}$. This inequality, together with (3.3), implies that $\left|\operatorname{Re} f(w)+C-\lambda^{\prime}\right| \leqq \varepsilon / 2$ for all $w \in V_{s}$. Therefore the horizontal oscillation of $f\left(V_{s}\right)$ is less than $\varepsilon$.

Let $S_{0}$ be the component of $S-f\left(V_{s_{0}}\right)$ whose boundary contains $+\infty$. Let $S_{1}=\{z \mid 0<\operatorname{Re} z<+\infty, 0<y<1\}$. Let $g$ be the 
one-to-one conformal map of $S_{0}$ onto $S_{1}$ which makes the upper and lower edges of $S_{0}$ correspond, respectively, to the upper and lower edges of $S_{1}$. Since $g$ is differentiable at $+\infty$, there is a real constant $C_{0}$ such that $g(z)-z \rightarrow C_{0}$ as $z \rightarrow+\infty$. Therefore, since the horizontal oscillation of $f\left(V_{s}\right)$ tends to zero as $s \rightarrow+\infty$, the same is true for $g\left(f\left(V_{s}\right)\right)$. Therefore

$$
\operatorname{Re} g(f(w))-\lambda\left(V_{s_{0}}, V_{s} ; H_{0}, H_{1}\right) \rightarrow 0 \quad \text { as } \quad s \rightarrow+\infty, w \in V_{s} .
$$

\section{Therefore}

$$
\operatorname{Re} f(w)+C_{0}-\lambda\left(V_{s_{0}}, V_{s} ; H_{0}, H_{1}\right) \rightarrow 0 \quad \text { as } \quad s \rightarrow+\infty, w \in V_{s} .
$$

This completes the proof of Theorem 1.

Condition I is actually necessary and sufficient for (3.2). Both conditions are related to the oscillation

$$
\omega(s)=\sup \left\{\operatorname{Re} f\left(w_{2}\right)-\operatorname{Re} f\left(w_{1}\right) \mid w_{1}, w_{2} \in V_{s}\right\} .
$$

We have the

Co rolla ry. The following statements are equivalent:

(i) The cross cuts $\left\{V_{s}\right\}$ satisfy Condition $I$.

(ii) The oscillation $\omega(s) \rightarrow 0$ as $s \rightarrow+\infty$.

(iii) There is a constant $C$ such that Equation (3.2) holds.

The proof of Theorem 1 shows that (i) implies (ii), and that (ii) implies (iii). Clearly, (iii) implies (ii). To prove that (ii) implies (i) we pass to the image quadrilaterals in $S$ by means of $f$ and verify the inequality that corresponds to (3.1) there. This inequality is evident there because these quadrilaterals differ from rectangles only by the horizontal oscillation of their vertical sides, and these oscillations tend to zero.

4. Behavior of $\operatorname{Im} f$. In this section we give a condition which will be useful for proving that $H_{t}$ is asymptotic to the level line

$$
\{w \in R \mid \operatorname{Im} f(w)=t\} .
$$

Condition II. Given $0<t<1$ and $\varepsilon>0$, there is an $a=$ $a(t, \varepsilon)>0, M=M(t), s_{I I}=s_{I I}(t, \varepsilon)$, and a function $d(s)>s$, where $d(s)$ also depends on $t$ and $\varepsilon$, such that if $s>s_{I I}$ then

$$
\begin{aligned}
& \frac{\lambda\left(H_{0}, H_{t} ; V_{s}, V_{d(s)}\right)}{\lambda\left(H_{0}, H_{1} ; V_{s}, V_{d(s)}\right)} \geqq t-\varepsilon, \\
& \frac{\lambda\left(H_{t}, H_{1} ; V_{s}, V_{d(s)}\right)}{\lambda\left(H_{0}, H_{1} ; V_{s}, V_{d(s)}\right)} \geqq 1-t-\varepsilon,
\end{aligned}
$$

and $a(t, \varepsilon) \leqq \lambda\left(V_{s}, V_{d(s)} ; H_{0}, H_{1}\right) \leqq M(t)$.

Th e o r e 2, Suppose Conditions I and II hold. Suppose 


$$
\lambda\left(V_{s_{1}}, V_{s_{2}} ; H_{0}, H_{1}\right)
$$

is a continuous function of $s_{1}$ and $s_{2}$. Let $0<t<1$. Then

$$
\lim _{s \rightarrow+\infty}[\operatorname{Im} f(w)-t]=0, \quad\left(w \in V_{s} \cap H_{t}\right) .
$$

Proof. Let $0<t<1$ and $\varepsilon>0$ be given. Use the $M=M(t)$, given by Condition II, in Lemma 4 to determine $\delta_{4}=\delta_{4}(\varepsilon / 2)$. Now apply Condition II for $0<t<1$ and $\delta_{4}>0$ to determine $a=a\left(t, \delta_{4}\right), s_{I I}=$ $s_{I I}\left(t, \delta_{4}\right)$, and $d(s)$. Determine $\delta_{3}=\delta_{3}(a / 4)$ from Lemma 3. Determine $s_{I}=s_{I}\left(\delta_{3}\right)$ from Condition I. Determine $\delta_{1}=\delta_{1}(a, \varepsilon / 2)$ from Lemma 1. By Theorem 1 we can choose $\sigma$ so large that for all $w \in V_{s}$ and all $s>\sigma$

$$
\left|\operatorname{Re} f(w)-C-\lambda\left(V_{s_{0}}, V_{s} ; H_{0}, H_{1}\right)\right|<\frac{\delta_{1}}{2} .
$$

Let $\sigma_{0}=\max \left(s_{I}, s_{I I}, \sigma\right)$ and choose $\sigma_{1}>\sigma_{0}$ so that

$$
\lambda\left(V_{\sigma_{0}}, V_{\sigma_{1}} ; H_{0}, H_{1}\right)>a / 2 \text {. }
$$

We shall show that $w \in V_{s} \cap H_{t}$ implies $|\operatorname{Im} f(w)-t|\left\langle\varepsilon\right.$ for all $s>\sigma_{1}$.

Suppose then that $s>\sigma_{1}$ and $w \in V_{s}$. Choose, by the continuity hypothesis, an $s_{1}<s$ so that $\lambda\left(V_{s_{1}}, V_{s} ; H_{0}, H_{1}\right)=a / 2$; note that $s_{1}>\sigma_{0}$. Let $Q_{0}=Q\left(V_{s_{1}}, V_{d\left(s_{1}\right)} ; H_{0}, H_{1}\right)$ and note that $A \equiv$ $\lambda\left(V_{s_{1}}, V_{d\left(s_{1}\right)} ; H_{0}, H_{1}\right) \geqq a$ according to Condition II. Let $\psi$ be the quadrilateral map of $Q_{0}$ onto $Q_{1}=Q(x=0, x=A ; y=0, y=1)$. Condition I supplies an extremal distance inequality for the components of $Q_{0}-V_{s}$, the inequality can be transferred to $Q_{1}$ via $\psi$, and when Lemma 3 is applied in $Q_{1}$ one obtains

$$
\left|\operatorname{Re} \psi(w)-\frac{a}{2}\right| \leqq \frac{a}{4}, \quad \text { for } w \in V_{s} .
$$

Now (4.1) and (4.2) hold when $s$ and $\varepsilon$ are replaced by $s_{1}$ and $\delta_{4}$; these inequalities can be transferred to $Q_{1}$, and when Lemma 4 is applied we obtain

$$
|\operatorname{Im} \psi(w)-t| \leqq \frac{\varepsilon}{2} \quad \text { for } w \in V_{s} \cap H_{t}
$$

Consider $g=f^{\prime} \circ \psi^{-1}$, a quadrilateral map of $Q_{1}$ onto $f\left(Q_{0}\right)$. Equation (4.3) implies that the horizontal oscillations of $f\left(V_{s_{1}}\right)$ and $f\left(V_{d\left(s_{1}\right)}\right)$ are no greater than $\delta_{1}=\delta_{1}(a, \varepsilon / 2)$. We apply Lemma 1 to $g-b$, where $b=\lambda\left(V_{s_{0}}, V_{s_{1}} ; H_{0}, H_{1}\right)+C$, and obtain

$$
|(g(z)-b)-z|<\frac{\varepsilon}{2}
$$

whenever $|\operatorname{Re} z-(a / 2)|<a / 4$. 
From (4.4) it follows that $z$ in (4.6) can be replaced by $\psi(w)$; this gives $|f(w)-\psi(w)-b|<\varepsilon / 2$, and hence $|\operatorname{Im} f(w)-\operatorname{Im} \psi(w)|<\varepsilon / 2$. When this last inequality is combined with (4.5) the desired result $|\operatorname{Im} f(w)-t|<\varepsilon$ is obtained.

An examination of the preceding proof leads to the following

$\mathrm{R}$ e $\mathrm{m}$ a $\mathrm{rk}$. If the parameters $M, a, d(s)$, and $s_{I I}$ in Condition II are independent of $t$ then the conclusion of Theorem 2,

$$
\lim _{s \rightarrow+\infty}[\operatorname{Im} f(w)-t]=0 \quad\left(w \in V_{s} \cap H_{t}\right),
$$

holds uniformly for all $t \in(0,1)$.

\section{Applications to general strip domains}

Theorems 1 and 2 of Chapter I will now be applied to the special case that $R$ is a strip domain and $\left\{V_{s}\right\}$ are the Ahlfors cross cuts $\left\{\vartheta_{u}\right\}$. Theorems 3 and 4 concern the real part of $f$ and the Ahlfors integral $\int d u / \vartheta(u)$. Theorem 5 gives a condition for the imaginary part of $f$ to behave approximately linearly on each $\vartheta_{u}$. It is of interest to compare these results with Theorem 8 in Chapter IV which deals with the Ahlfors integral and linearity of $\operatorname{Im} f$ on $\vartheta_{u}$ for strip domains with smooth boundaries. Theorem 6 is an application to the angular derivative problem.

5. Strip domains. The general situation described in Section 1 applies when $R$ is a strip domain. This means that $R$ is a simply connected region in the $w=u+i v$ plane, and that there is an open arc $\tau \mapsto w(\tau)$, $0<\tau<+\infty$, in $R$ with $\lim _{\tau \rightarrow+\infty} \operatorname{Re} w(\tau)=+\infty$; this arc determines the prime end $\zeta$. It is known (Ahlfors [1]) that one can then choose a family $\left\{\vartheta_{u}\right\}_{u \geqq u_{0}}$ of cross cuts of $R$ with the following properties: (i) each $\vartheta_{u}$ lies on a vertical line with real part $u$, (ii) each $\vartheta_{u}$ separates $\zeta$ from the prime end $\zeta_{0}=f^{-1}(-\infty)$, (iii) if $u_{0} \leqq u_{1}<u_{2}$ then $\vartheta_{u_{2}}$ separates $\vartheta_{u_{1}}$ from $\zeta$, and (iv) if $\vartheta(u)$ is the Euclidean length of $\vartheta_{u}$ then $\vartheta(u)$ is a measurable function of $u$ (note that we allow $\vartheta(u)=+\infty$ ). The cross cuts $\left\{\vartheta_{u}\right\}_{u \geqq u_{0}}$ qualify as a special case of the general cross cuts $\left\{V_{s}\right\}_{s} \geqq s_{n}$ of Section 1 (cf. Figure 2). A strip domain consists of such an $R$ together with the associated quantities $\zeta_{0}, \zeta,\left\{\vartheta_{u}\right\}_{u \geqq u_{0}}$

The integral $\int d u / \vartheta(u)$ played an inportant role in Ahlfors [1]. It is well known that this integral provides a lower bound for the extremal distance between $\vartheta_{u_{1}}$ and $\vartheta_{u_{2}}$ (see, for example, $[2$, p. 56] or $[11$, p. 665]): 


$$
\int_{u_{1}}^{u_{2}} \frac{d u}{\vartheta(u)} \leqq \lambda\left(\vartheta_{u_{1}}, \vartheta_{u_{2}} ; H_{0}, H_{1}\right)
$$

Define the error term $E\left(u_{1}, u_{2}\right)$ in this estimate by

$$
\lambda\left(\vartheta_{u_{1}}, \vartheta_{u_{2}} ; H_{0}, H_{1}\right)=\int_{u_{1}}^{u_{2}} \frac{d u}{\vartheta(u)}+E\left(u_{1}, u_{2}\right) \quad\left(u_{1}<u_{2}\right) .
$$

Note that $E\left(u_{1}, u_{2}\right) \geqq 0$. In Sections 6 and 7 we investigate the consequences that follow when $E\left(u_{1}, u_{2}\right)$ is unformly bounded.

6. Boundedness of $E\left(u_{1}, u_{2}\right)$. Let $R$ be a strip domain. Suppose that $E\left(u_{1}, u_{2}\right)$ in Equation (5.2) is bounded for all $u_{1}>u_{0}$. It follows that $E\left(u_{1}, u_{2}\right) \rightarrow 0$ as $u_{1} \rightarrow+\infty$. Indeed, since extremal distance is subadditive (i.e., $\lambda\left(\vartheta_{u_{1}}, \vartheta_{u} ; H_{0}, H_{1}\right)+\lambda\left(\vartheta_{u}, \vartheta_{u_{2}} ; H_{0}, H_{1}\right) \leqq$ $\left.\lambda\left(\vartheta_{u_{1}}, \vartheta_{u_{2}} ; H_{0}, H_{1}\right)\right),(5.2)$ shows $E\left(u_{1}, u_{2}\right)$ is subadditive:

$$
E\left(u_{1}, u\right)+E\left(u, u_{2}\right) \leqq E\left(u_{1}, u_{2}\right) \quad\left(u_{1}<u<u_{2}\right) .
$$

Therefore $E\left(u_{1}, u\right)$ is an increasing function of $u$; let

$$
A=\lim _{u \rightarrow+\infty} E\left(u_{1}, u\right) \text {. }
$$

Let $u \rightarrow+\infty$ in (6.1) and obtain $A+\lim _{u \rightarrow+\infty} E\left(u, u_{2}\right) \leqq A$. Therefore

$$
\lim _{u \rightarrow+\infty} E\left(u, u_{2}\right)=0 \quad\left(u<u_{2}\right)
$$

as asserted.

Now consider Condition I of Section 3. Equations (5.2) and (6.2) show immediately that Condition $I$ is satisfied in this case. Therefore Theorem 1 can be applied; the result can be stated as follows:

Th e or e m 3. Let $R$ be a strip domain. Suppose the error function $E\left(u_{1}, u_{2}\right)$ of Equation (5.2) is uniformly bounded for all $u_{1}>u_{0}$. Then for all $u>u_{0}$ and all $w \in \vartheta_{u}$ we have

$$
\operatorname{Re} f(w)=\int_{u_{0}}^{u} \frac{d u}{\vartheta(u)}+C+E(u)
$$

where $C$ is a real constant and $\lim _{u \rightarrow+\infty} E(u)=0$.

Theorem 3 yields a short proof of a theorem of B. G. Eke [3, Theorem 2]. Let

$$
\underline{x}(u)=\min _{w \in \vartheta_{u}} \operatorname{Re} f(w), \quad \bar{x}(u)=\max _{w \in \vartheta_{u}} \operatorname{Re} f(w) .
$$


We shall prove:

Th e o r e m 4 (Eke [3]). Let $R$ be a strip domain. Then the quantities

$$
\underline{x}(u)-\int_{u_{0}}^{u} \frac{d u}{\vartheta(u)} \quad \text { and } \quad \bar{x}(u)-\int_{u_{0}}^{u} \frac{d u}{\vartheta(u)}
$$

tend to a common limit $\beta$ as $u \rightarrow+\infty$, where $-\infty<\beta \leqq+\infty$.

Proof. Ahlfors' Distortion Theorem [1] states that if $u_{2}>u_{1}>u_{0}$ and $\int_{u_{1}}^{u_{2}} d u / \vartheta(u)>2$ then $x\left(u_{2}\right)-\bar{x}\left(u_{1}\right) \geqq \int_{u_{1}}^{u_{2}} d u / \vartheta(u)-4$. An immediate consequence is that the two quantities in (6.4) are either simultaneously bounded or else tend simultaneously to $+\infty$ as $u \rightarrow+\infty$. The second alternative corresponds to $\beta=+\infty$ in Theorem 4 . If the first alternative holds, let $M$ be a bound for the two quantities. Then

$$
\begin{aligned}
\int_{u_{1}}^{u_{2}} \frac{d u}{\vartheta(u)} & \leqq \lambda\left(\vartheta_{u_{1}}, \vartheta_{u_{2}} ; H_{0}, H_{1}\right)=\lambda\left(f\left(\vartheta_{u_{1}}\right), f\left(\vartheta_{u_{2}}\right) ; y=0, y=1\right) \\
& \leqq \lambda\left(x=\underline{x}\left(u_{1}\right), x=\bar{x}\left(u_{2}\right) ; y=0, y=1\right)=\bar{x}\left(u_{2}\right)-\underline{x}\left(u_{1}\right) \\
& =\left[\bar{x}\left(u_{2}\right)-\int_{u_{0}}^{u_{2}} \frac{d u}{\vartheta(u)}\right]-\left[\underline{x}\left(u_{1}\right)-\int_{u_{0}}^{u_{1}} \frac{d u}{\vartheta(u)}\right]+\int_{u_{1}}^{u_{2}} \frac{d u}{\vartheta(u)} \\
& \leqq 2 M+\int_{u_{1}}^{u_{2}} \frac{d u}{\vartheta(u)}
\end{aligned}
$$

which shows that $E\left(u_{1}, u_{2}\right)$ of $(5.2)$ is uniformly bounded by $2 M$. Therefore Theorem 3 can be applied; it shows that the quantities (5.6) have a common finite limit $C$.

7. Approximately linear behavior of $\operatorname{Im} f$. We have seen (Theorem 3 ) that the boundedness of $E\left(u_{1}, u_{2}\right)$ implies that $\operatorname{Re} f(w)$ behaves approximately like $\int_{u_{0}}^{u} d u / \vartheta(u)+$ const. $\left(w \in \vartheta_{u}\right)$. Can conclusions be drawn concerning the behavior of $\operatorname{Im} f(w)$ under this hypothesis? The simplest behavior would be for $\operatorname{Im} f(u+i v)$ to be "approximately linear" in $v$ along $\vartheta_{u}$. That is, if $h_{t}(u)$ denotes the ordinate of the point on $\vartheta_{u}$ which divides $\vartheta_{u}$ into segments of lengths $t \vartheta(u)$ and $(1-t) \vartheta(u)$, the length of the lower segment being $t \vartheta(u)$, where $0<t<1$, then we say $\operatorname{Im} f$ is approximately linear on $\vartheta_{u}$ if $\operatorname{Im} f\left(u+i h_{t}(u)\right) \rightarrow t$ as $u \rightarrow+\infty$.

To provide a background for the next theorem we now describe an example in which $E\left(u_{1}, u_{2}\right)$ is bounded (and so $\operatorname{Re} f(u+i v)=$ const. + 
$\left.\int_{u_{0}}^{u} d u / \vartheta(u)+o(1)\right)$ and yet $\operatorname{Im} f$ is not approximately linear on $\vartheta_{u}$. Let $R^{\prime}$ be the parallel strip $\{w \mid-\infty<\operatorname{Re} w<+\infty, 0<\operatorname{Im} w<1\}$. Let $\left\{b_{n}\right\}_{n=1,2}, \ldots$ be a sequence of positive numbers such that $\sum b_{n}<\infty$. For each $n=1,2, \ldots$ consider the isosceles triangle with vertices at $n+2 i, n-b_{n}+i$, and $n+b_{n}+i$. Let $T_{n}$ be the interior of this triangle together with its horizontal base. Let $R=R^{\prime} \cup \cup_{n=1}^{\infty} T_{n}$, and consider $R$ as a strip domain with $\zeta_{0}=-\infty, u_{0}=0$ (see Section 5).

We have

$$
\int_{u_{1}}^{u_{2}} \frac{d u}{\vartheta(u)} \leqq \lambda\left(\vartheta_{u_{1}}, \vartheta_{u_{2}} ; H_{0}, H_{1}\right) \leqq u_{2}-u_{1}=\int_{u_{1}}^{u_{2}} \frac{d u}{\vartheta(u)}+\tilde{E}\left(u_{1}, u_{2}\right)
$$

where $\tilde{E}\left(u_{1}, u_{2}\right)=\int_{u_{1}}^{u_{2}}\left(1-\vartheta(u)^{-1}\right) d u$ is uniformly bounded for $u_{2}>$ $u_{1}>0$. Therefore $E\left(u_{1}, u_{2}\right)$ of $(5.2)$ is uniformly bounded. It will be shown that for each fixed $0<t<1$,

$$
\lim _{u \rightarrow+\infty} \operatorname{Im} f(u+i t)=t
$$

From (7.2) it follows that $\operatorname{Im} f$ is not approximately linear on $\vartheta_{u}$.

Note that (7.1) implies $\lambda\left(\vartheta_{u_{1}}, \vartheta_{u_{2}} ; H_{0}, H_{1}\right)=u_{2}-u_{1}+o(1)$ where $o(1) \rightarrow 0$ as $u_{2}>u_{1} \rightarrow+\infty$. In Theorem 6 we shall see that this condition implies (7.2). (It is also possible to prove (7.2) directly by means of the Poisson formula.)

8. Uniformly continuous boundary. The above example suggests that the hypothesis of bounded $E\left(u_{1}, u_{2}\right)$ may imply that $\operatorname{Im} f$ is approximately linear on $\vartheta_{u}$ in a sense of mean convergence rather than the pointwise sense. We shall not pursue that direction here; instead we present a condition on $R$ which, together with the boundedness of $E\left(u_{1}, u_{2}\right)$, does imply approximately linear behavior in the strict sense.

Let $\varphi_{+}$and $\varphi_{-}$be continuous real valued functions of a real variable, each with domain $(-\infty, \infty)$. Assume $\varphi_{+}>\varphi_{-}$. Let $R$ be the strip domain bounded by the graphs of $\varphi_{+}$and $\varphi_{-}$. If $\varphi_{+}$and $\varphi_{-}$are uniformly continuous we shall say that $R$ is a strip domain with uniformly continuous boundary. In that case define

$$
H_{t}=\left\{w=u+i v \mid v=t \varphi_{+}(u)+(1-t) \varphi_{-}(u)\right\} \quad(0<t<1) .
$$

Note that $H_{t}$ satisfies the requirements of Section 1. The approximately linear behavior of $\operatorname{Im} f$ on $\vartheta_{u}$ can now be expressed by (8.2) below.

Th e o r e m 5. Assume (i) $R$ is a strip domain with uniformly continuous boundary, (ii) there are positive constants $c, L$ such that $c<\vartheta(u)<L$ 
for all sufficiently large $u$, and (iii) ${ }^{1}$ any one of the conditions in the Corollary to Theorem 1 holds. Let $0<t<1$. Then

$$
\lim _{u \rightarrow+\infty}[\operatorname{Im} f(w)-t]=0 \quad\left(w \in H_{t} \cap \vartheta_{u}\right) .
$$

Proof. The first step of the proof will be to verify that Condition II of Section 4 is satisfied for $R$ when $H_{t}$ is defined by (8.1) and $\left\{V_{s}\right\}$ are the Ahlfors cross cuts $\left\{\vartheta_{u}\right\}$. Let $0<t<1$ and $\varepsilon>0$. Choose $\eta>0$ so that

$$
\eta \leqq \frac{c t}{2}, \quad \eta \leqq \frac{c(1-t)}{2}, \quad \text { and } \quad \eta \leqq \frac{\varepsilon c}{3}
$$

Choose $d_{0}>0$ so that $d_{0}<1$ and

$$
\left|\varphi_{ \pm}(u)-\varphi_{ \pm}\left(u^{\prime}\right)\right| \leqq \eta \quad \text { whenever }\left|u-u^{\prime}\right|<d_{0} .
$$

Define $d(u)$ by $d(u)=u+d_{0}$. Choose $s_{I I}$ so that hypothesis (ii) of the theorem holds for $u \geq s_{I I}$. With these choices we shall show that (4.1) and (4.2) of Condition II hold. For $u \geqq s_{I I}$ define

$$
\vartheta^{*}=\min _{u \leqq u^{\prime} \leqq d(u)}\left[t \varphi_{+}\left(u^{\prime}\right)+(1-t) \varphi_{-}\left(u^{\prime}\right)\right]-\max _{u \leqq u^{\prime} \leqq d(u)} \varphi_{-}\left(u^{\prime}\right) .
$$

Then $\vartheta^{*} \geqq t \vartheta_{\min }-\eta \geqq t c-\eta \geqq 0$, where

$$
\vartheta_{\min }=\min \left\{\vartheta\left(u^{\prime}\right) \mid u \leqq u^{\prime} \leqq d(u)\right\} .
$$

We can therefore estimate $\lambda\left(\vartheta_{u}, \vartheta_{d(u)} ; H_{0}, H_{t}\right)$ from above by inscribing a rectangle of width $\vartheta^{*}$; we can estimate $\lambda\left(\vartheta_{u}, \vartheta_{d(u)} ; H_{0}, H_{1}\right)$ from below by the usual lower bound (5.1). These estimates lead to

$$
\begin{aligned}
& \lambda\left(\vartheta_{u}, \vartheta_{d(u)} ; H_{0}, H_{t}\right) \leqq \frac{d(u)-u}{\vartheta *} \leqq \frac{d_{0}}{t \vartheta_{\min }-\eta}, \\
& \lambda\left(\vartheta_{u}, \vartheta_{d(u)} ; H_{0}, H_{1}\right) \geqq \int_{u}^{d(u)} \frac{d u}{\vartheta(u)} \geqq \frac{d_{0}}{\vartheta_{\max }},
\end{aligned}
$$

where $\vartheta_{\max }=\max \left\{\vartheta\left(u^{\prime}\right) \mid u \leqq u^{\prime} \leqq d(u)\right\}$. Clearly $\left(\vartheta_{\max }-\vartheta_{\min }\right) / \vartheta_{\max }$ $\leqq 2 \eta / c$, so $1-(2 \eta / c) \leqq \vartheta_{\min } / \vartheta_{\max }$. We use this together with (8.5) and (8.6) to obtain

$$
\begin{aligned}
\frac{\lambda\left(H_{0}, H_{t} ; \vartheta_{u}, \vartheta_{d(u)}\right)}{\lambda\left(H_{0}, H_{1} ; \vartheta_{u}, \vartheta_{d(u)}\right)} & \geqq t \frac{\vartheta_{\min }}{\vartheta_{\max }}\left(1-\frac{\eta}{t \vartheta_{\min }}\right) \\
& \geqq t\left(1-\frac{2 \eta}{c}\right)\left(1-\frac{\eta}{t c}\right) \geqq t-\frac{3 \eta}{c} \geqq t-\varepsilon .
\end{aligned}
$$

1 This will be the case if, for example, $E\left(u_{1}, u_{2}\right)$ in (5.2) is uniformly bounded (see Theorem 3). 
Therefore (4.1) is satisfied. In the same way (4.2) can be verified. To complete the verification of Condition II the bounds $a(t, \varepsilon)$ and $M(t)$ must be exhibited. The upper bound $M(t)=2 / c$ can be obtained in the same way that (8.5) was derived,

$$
\lambda\left(\vartheta_{u}, \vartheta_{d(u)} ; H_{0}, H_{1}\right) \leqq \frac{d_{0}}{c-\eta} \leqq \frac{2}{c},
$$

the last inequality resulting from our choices $d_{0} \leqq 1$ and $\eta \leqq c / 2$. The lower bound $a(t, \varepsilon)=d_{0} / L>0$ follows directly from (8.6). The verification of Condition II is now complete.

Hypothesis (iii) of the present theorem implies that Condition $I$ is satisfied (see the Corollary of Theorem 1). We have already proved that Condition II is satisfied. In order to apply Theorem 2 it only remains to prove that $\lambda\left(\vartheta_{u_{1}}, \vartheta_{u_{2}} ; H_{0}, H_{1}\right)$ is continuous in $u_{1}$ and in $u_{2}$. Although this continuity property need not hold for an arbitrary strip domain, it does hold for a strip domain bounded by the graphs of continuous functions. In this latter case the property amounts to the convergence of a suitably normalized family of conformal mappings onto rectangles, and can be proved by the Carathéodory kernel theorem. We shall omit the details of the proof. The conclusion (8.2) of the present theorem now follows immediately from Theorem 2.

$\mathrm{R}$ e $\mathrm{m}$ a $\mathrm{rk}$. An examination of the proof shows that (8.2) holds uniformly for all $t$ with $|t-1 / 2| \leqq$ const. $<1 / 2 \quad$ (cf. the Remark following Theorem 2).

9. An application to the angular derivative problem. Let $R$ be a strip domain in the $w=u+i v$ plane. In addition to the notations $\zeta, \vartheta_{u}, S, f$ introduced in Sections 1 and 5, also define $g=f^{-1}: S \rightarrow R$. The angular derivative $C$ at $\zeta$ may be defined as

$$
\lim _{\substack{x \rightarrow+\infty \\ \delta<y<1-\delta}}[z-g(z)]=C
$$

provided the limit exists for every $0<\delta<1 / 2$, and $C \neq \infty$.

The angular derivative problem is to find geometric properties of $R$ which are necessary and sufficient for the existence of an angular derivative at $\zeta$. Progress on this famous problem has been made by numerous mathematicians (see [12] for a survey of results prior to 1955; subsequent work has been done in [4], [5], [6], [10], [19], [20]) but it still remains unsolved.

Another standard, and essentially equivalent, definition of the angular derivative will be more useful for our purposes. By performing a vertical translation of $R$ one can require that $C$ be real. Then, since $\operatorname{Im} g(z)-y$ 
$\rightarrow 0$ in $\{\delta<y<1-\delta\}$ as $x \rightarrow+\infty$, it follows that $R$ must contain a half strip

$$
R_{\delta}=\left\{w \mid u \geqq U_{\delta}, \delta<v<1-\delta\right\}
$$

for each $0<\delta<1 / 2$. $\zeta$ must be the prime end at $+\infty$ which is accessible along the ray $\left\{u \geqq U_{\delta}, v=1 / 2\right\}$. Furthermore, there is a $u_{0}$ such that $\vartheta_{u}$ intersects the line $\{v=1 / 2\}$ for all $u \geqq u_{0}$. From now on we adopt the following definition of angular derivative:

$\mathrm{D}$ efinition. A strip domain $R$ in the w-plane is said to possess an angular derivative $C$ at $\zeta$ provided, for every $0<\delta<1 / 2$,

$$
R \text { contains a half-strip } R_{\delta} \text { of the form (9.1), }
$$

and

$$
\lim _{\operatorname{Re} w \rightarrow+\infty}[f(w)-w]=C \neq \pm \infty \quad\left(w \in R_{\delta}\right) .
$$

Note that $C$ in (9.3) is necessarily real. Condition (9.3) is often replaced by the pair of conditions:

$$
\begin{gathered}
\lim _{\operatorname{Re} w \rightarrow+\infty}[\operatorname{Re} f(w)-\operatorname{Re} w]=C \neq \pm \infty \quad\left(w \in R_{\delta}\right) \\
\lim _{\operatorname{Re} w \rightarrow+\infty}[\operatorname{Im} f(w)-\operatorname{Im} w]=0 \quad\left(w \in R_{\delta}\right) .
\end{gathered}
$$

Condition $(9.3 \mathrm{~b})$ is traditionally referred to as semiconformality at $\zeta$.

In Theorem 6 we present an extremal length property of $R$ which is necessary and sufficient for the existence of an angular derivative at $\zeta$. This result should not be considered as a solution to the classical angular derivative problem because extremal length is not a geometric property in the classical (Euclidean) sense. The result does indicate, however, that extremal length may be a fruitful tool for future progress on the problem.

Th e o r e m $6{ }^{1}$. Let $R$ be a strip domain. The following properties are necessary and sufficient for the existence of an angular derivative at $\zeta$ :

(i) $R$ contains a half-strip $R_{\delta}$ of the form (9.1) for every $0<\delta<1 / 2$,

(ii) $\lambda\left(\vartheta_{u_{1}}, \vartheta_{u_{2}} ; H_{0}, H_{1}\right)=u_{2}-u_{1}+E\left(u_{1}, u_{2}\right)$ where $E\left(u_{1}, u_{2}\right) \rightarrow 0$ as $u_{1} \rightarrow+\infty \quad\left(u_{1}<u_{2}\right)$.

Proof. Suppose the angular derivative exists. Then (i) holds. It is well known (for example, Warschawski [19, Theorem 1 b and Theorem 2]) that the semiconformality implies

$$
\tau(u)=\sup \left\{|\operatorname{Re} f(u)-\operatorname{Re} f(w)| \mid w \in \vartheta_{u}\right\} \rightarrow 0
$$

${ }^{1} \mathrm{~K}$. Oikawa has informed us that he and J. Jenkins have independently obtained. this same theorem in joint work in progress. 
as $u \rightarrow+\infty$. This fact together with (9.3a) shows that $f\left(\vartheta_{u}\right)$ "approaches" the segment $\{x=u+C, 0<y<1\}$. If $\varepsilon>0$ then for all sufficiently large $u_{1}$ with $u_{1}+\varepsilon<u_{2}-\varepsilon$,

$$
\begin{aligned}
Q\left(x=u_{1}+C+\varepsilon, x\right. & \left.=u_{2}+C-\varepsilon ; y=0, y=1\right) \subset Q\left(f\left(\vartheta_{u_{1}}\right), f\left(\vartheta_{u_{2}}\right) ; y=0, y=1\right) \\
& \subset Q\left(x=u_{1}+C-\varepsilon, x=u_{2}+C+\varepsilon ; y=0, y=1\right) .
\end{aligned}
$$

Therefore

$$
\begin{aligned}
u_{2}-u_{1}-2 \varepsilon & \leqq \lambda\left(f\left(\vartheta_{u_{1}}\right), f\left(\vartheta_{u_{2}}\right) ; y=0, y=1\right) \\
& =\lambda\left(\vartheta_{u_{1}}, \vartheta_{u_{2}} ; H_{0}, H_{1}\right) \leqq u_{2}-u_{1}+2 \varepsilon .
\end{aligned}
$$

If $u_{1}+\varepsilon \geqq u_{2}-\varepsilon$ then $u_{2}-u_{1}-2 \varepsilon \leqq 0$ and these last inequalities still hold. Thus (ii) holds.

Conversely, suppose (i) and (ii) hold. Condition I of Section 3 follows at once from (ii). Thus Theorem 1 is applicable and it yields (a stronger version of) (9.3a). It follows from (9.3a) that $f^{\prime}(w) \rightarrow 1$ as $u \rightarrow+\infty$, uniformly in any $R_{\delta}$, as can be easily seen from the representation

$$
f^{\prime}(a)=\frac{1}{\pi i} \int_{\left|w-a_{0}\right|=r} \operatorname{Re} f(w) \frac{d w}{(w-a)^{2}} \quad\left(\left|a-a_{0}\right|<r\right)
$$

which is an immediate consequence of Schwarz's formula and is valid as soon as $f$ is analytic on $\left\{\left|w-a_{0}\right| \leqq r\right\}$. Therefore $f(u+(1-\delta) i)-$ $f(u+\delta i) \rightarrow(1-2 \delta) i$ as $u \rightarrow+\infty$. Therefore, since $0<\operatorname{Im} f(w)<1$,

$$
\begin{gathered}
0 \leqq \limsup _{u \rightarrow+\infty} \operatorname{Im} f(u+\delta i) \leqq 2 \delta, \\
1-2 \delta \leqq \liminf _{u \rightarrow+\infty} \operatorname{Im} f(u+(1-\delta) i) \leqq 1 .
\end{gathered}
$$

This shows that the bounded harmonic function $p(w)=\operatorname{Im} f(w)-$ $\operatorname{Im} w$ satisfies

$$
\limsup _{u \rightarrow+\infty}|p(u+\delta i)| \leqq \delta \quad \text { and } \quad \limsup _{u \rightarrow+\infty}|p(u+(1-\delta) i)| \leqq \delta .
$$

Hence $\lim \sup |p(w)| \leqq \delta$ for $w \in R_{\delta}$ and $\operatorname{Re} w \rightarrow+\infty$. Choose $\delta^{\prime}$ so that $0<\delta^{\prime}<\delta$. Then $\lim \sup |p(w)| \leqq \delta^{\prime} \quad$ for $w \in R_{\delta}$ and $\operatorname{Re} w \rightarrow+\infty$. Since $\delta^{\prime}$ is arbitrary, $p(w) \rightarrow 0$ for $w \in R_{\delta}, \operatorname{Re} w \rightarrow+\infty$. This proves $(9.3 \mathrm{~b})$, and completes the proof of Theorem 5 .

10. An angular derivative criterion. Theorem 6 reduces the angular derivative problem to one of estimating extremal distances. As an illustration we present the following example.

Let $\left\{u_{j}\right\},\left\{v_{j}\right\},\left\{v_{j}^{\prime}\right\}$ be sequences of real numbers such that $u_{j} \nearrow+\infty$ and $v_{j}<1 / 2<v_{j}^{\prime}$. Define 


$$
L_{j}=\left\{u_{j}+i v \mid v \leqq v_{j}\right\}, \quad L_{j}^{\prime}=\left\{u_{j}+i v \mid v \geqq v_{j}^{\prime}\right\}
$$

and let $P$ be the strip domain $C-\cup_{j}\left(L_{j} \cup L_{j}^{\prime}\right)$. We shall make use of the parameters

$$
\Theta_{j}=\min \left(v_{j-1}^{\prime}, v_{j}^{\prime}\right)-\max \left(v_{j-1}, v_{j}\right), \quad d_{j}=u_{j}-u_{j-1}
$$

to give a lower bound for $\lambda\left(\vartheta_{u_{m}}, \vartheta_{u_{n}} ; H_{0}, H_{1}\right),(m<n)$. Write $P=$ $P\left(\left\{\Theta_{j}\right\},\left\{d_{j}\right\}\right)$ to indicate that $P$ is a domain with these parameters.

The lower bound will be obtained by calculating the appropriate lengths and areas with a special metric $\varrho(w)|d w|$. Let $T_{j}$ be the rectangle

$\left\{u+i v \mid u_{j-1} \leqq u \leqq u_{j}, \max \left(v_{j-1}, v_{j}\right)-d_{j} \leqq v \leqq \min \left(v_{j-1}^{\prime}, v_{j}^{\prime}\right)+d_{j}\right\}$. Set $\varrho(w)=1$ in $\bigcup_{m+1}^{n} T_{j}$, and $\varrho(w)=0$ elsewhere. Any are in $P$ which joins $\vartheta_{u_{m}}$ to $\vartheta_{u_{n}}$ has $\varrho$-length at least $\sum_{j=m+1}^{n} d_{j}=u_{m}-u_{n}$. The $\varrho$-area is $\sum_{j=m+1}^{n}\left(\Theta_{j} d_{j}+2 d_{j}^{2}\right)$. Therefore

$$
\lambda\left(\vartheta_{u_{m}}, \vartheta_{u_{n}} ; H_{0}, H_{1}\right) \geqq \frac{\left(u_{m}-u_{n}\right)^{2}}{\sum_{j=m+1}^{n}\left(\Theta_{j} d_{j}+2 d_{j}^{2}\right)}=u_{m}-u_{n}-e+\frac{e^{2}}{u_{m}-u_{n}+e}
$$

where $e=e(m, n)=\sum_{j=m+1}^{n}\left[\left(\Theta_{j}-1\right) d_{j}+2 d_{j}^{2}\right]$. We shall use the lower bound (10.1) to prove the following statement, which can also be obtained from a theorem of J. Lelong-Ferrand [12, Théorème (VI. 19c) p. 214]:

Let $R_{0} \subset R \subset P=P\left(\left\{\Theta_{j}\right\},\left\{d_{j}\right\}\right)$ be strip domains. Suppose $R_{0}$ has an angular derivative at $+\infty$. If the series

$$
\sum_{j=1}^{\infty}\left(\Theta_{j}-1\right) d_{j} \quad \text { and } \quad \sum_{j=1}^{\infty} d_{j}^{2}
$$

converge then $R$ has an angular derivative at $+\infty$.

Proof. By Theorem 6 and the comparison principle for extremal length we obtain the upper estimate

$$
\lambda_{R}\left(\vartheta_{u^{\prime}}, \vartheta_{u^{\prime \prime}} ; H_{0}, H_{1}\right) \leqq \lambda_{R_{0}}\left(\vartheta_{u^{\prime}}, \vartheta_{u^{\prime \prime}} ; H_{0}, H_{1}\right)=u^{\prime \prime}-u^{\prime}+o(1)
$$

where $o(1) \rightarrow 0$ as $u^{\prime \prime}>u^{\prime} \rightarrow+\infty$. To obtain a similar lower estimate for $\lambda_{R}$ of the form $u^{\prime \prime}-u^{\prime}+o(1)$ we use

$$
\begin{aligned}
& \lambda_{R}\left(\vartheta_{u^{\prime}}, \vartheta_{u^{\prime \prime}} ; H_{0}, H_{1}\right)-\left(u^{\prime \prime}-u^{\prime}\right) \\
& \geqq \lambda_{P}\left(\vartheta_{u^{\prime}}, \vartheta_{u^{\prime \prime}} ; H_{0}, H_{1}\right)-\left(u^{\prime \prime}-u^{\prime}\right)=E\left(u^{\prime}, u^{\prime \prime}\right),
\end{aligned}
$$

and show that $E\left(u^{\prime}, u^{\prime \prime}\right) \geqq o(1)$ as $u^{\prime \prime}>u^{\prime} \rightarrow+\infty$.

Let $\varepsilon>0$. There is an $\eta>0$ such that $0<u^{\prime \prime}-u^{\prime}<\eta$ implies 
$\left|E\left(u^{\prime}, u^{\prime \prime}\right)\right|<\varepsilon$ for all $u^{\prime}>u_{0}$. We may therefore assume that $u^{\prime \prime}-u^{\prime}$ $\geqq \eta$ and then prove $E\left(u^{\prime}, u^{\prime \prime}\right)>-\varepsilon$ for sufficiently large $u^{\prime}$. Let $\left[u_{m}, u_{n}\right]$ be the largest interval, formed from the sequence $\left\{u_{j}\right\}$, which is contained in $\left[u^{\prime}, u^{\prime \prime}\right]$. If $u^{\prime}$ is sufficiently large then $m \neq n$ and the term $e=e(m, n)$ in (10.1) will satisfy $|e|<\eta / 2$. Therefore the term $e^{2} /\left(u_{m}-u_{n}+e\right)$ in (10.1) will be positive and can be omitted. The resulting (10.1) and the comparison principle yield

$$
E\left(u^{\prime}, u^{\prime \prime}\right) \geqq\left(u_{m}-u_{n}\right)-\left(u^{\prime \prime}-u^{\prime}\right)-e \geqq-d_{m}-d_{n+1}-e .
$$

This shows that $E\left(u^{\prime}, u^{\prime \prime}\right) \geqq-\varepsilon$ if $u^{\prime}$ is taken sufficiently large.

These upper and lower estimates prove that $\lambda_{R}\left(\vartheta_{u^{\prime}}, \vartheta_{u^{\prime \prime}} ; H_{0}, H_{1}\right)=$ $u^{\prime \prime}-u^{\prime}+o(1)$. Theorem 6 shows that $R$ has an angular derivative at $+\infty$.

\section{Estimates of extremal distance}

In this chapter we return to the general situation of $\S 1$. We require that the grid lines $\left\{V_{s}\right\},\left\{H_{t}\right\}$ be given explicitly as the image under a differentiable mapping of a rectangular coordinate grid in the plane. In this case there is a useful method (Theorem 7) for estimating the extremal distances $\lambda\left(V_{s_{1}}, V_{s_{2}} ; H_{t_{1}}, H_{t_{2}}\right)$. This method will be used in Chapter IV in order to apply Theorems 1 and 2 to special regions.

11. Definitions and notations. The notations $R,\left\{V_{s}\right\}_{s \geqq s_{0}},\left\{H_{t}\right\}_{0 \leqq t \leqq 1}$ have the meanings of $\S 1$. We now impose further special conditions.

Assume $R$ is a plane region. Assume that $H_{0}$ and $H_{1}$ are smooth curves on the border of $R$. Assume that each cross cut $V_{s}$ can be extended so that it possesses endpoints on $H_{0}$ and $H_{1}$; from now on $V_{s}$ will denote the extended cross cut. Assume that $\boldsymbol{c}=\boldsymbol{c}(s, t)$ is a homeomorphic mapping of the closed half-strip

$$
\left\{s+i t \mid s_{0} \leqq s<+\infty, 0 \leqq t \leqq 1\right\}
$$

into $R \cup H_{0} \cup H_{1}$. Assume that $c$ maps each vertical segment

$$
\{s+i t \mid s \text { fixed, } 0 \leqq t \leqq 1\}
$$

onto $V_{s}$, and each horizontal ray

$$
\left\{s+i t \mid s_{0} \leqq s<+\infty, t \text { fixed }\right\}
$$

onto $H_{t}$. Then each $V_{s}$ can be parametrized by $t \mapsto c(s, t)$, and each $H_{t}$ can be parametrized by $s \mapsto \boldsymbol{c}(s, t)$. For simplicity we require that $c(s, t)$ be continuously differentiable and that its Jacobian determinant 
$J(s, t)$ be positive for $s_{0}<s<+\infty, 0<t<1$. For later reference we summarize all these conditions by:

$$
\begin{aligned}
& V_{s}: t \mapsto V_{s}(t)=c(s, t), \quad 0 \leqq t \leqq 1, \\
& H_{t}: s \mapsto H_{t}(s)=c(s, t), \quad s_{0} \leqq s<+\infty, \\
& J(s, t)>0 \quad\left(s_{0}<s<+\infty, 0<t<1\right) .
\end{aligned}
$$

12. An extremal distance estimate. Let $R$ be a region as described in $\S 11$. The following theorem provides upper and lower estimates for extremal distances in the quadrilaterals determined by $\left\{V_{s}\right\}$ and $\left\{H_{t}\right\}$.

The o r e m 7. Assume that $\boldsymbol{c}(s, t)$ is a mapping with the properties (11.1)-(11.3). For $s_{0}<s_{1}<s_{2}$ and $0 \leqq t_{1}<t_{2} \leqq 1$ define

$$
k(s)=\int_{t_{1}}^{t_{2}} J^{-1}(s, t)\left|\frac{\partial}{\partial t} c(s, t)\right|^{2} d t
$$

and

$$
l(t)=\int_{s_{1}}^{s_{2}} J^{-1}(s, t)\left|\frac{\partial}{\partial s} c(s, t)\right|^{2} d s .
$$

Then

$$
\int_{s_{1}}^{s_{2}} \frac{d s}{k(s)} \leqq \lambda\left(V_{s_{1}}, V_{s_{2}} ; H_{t_{1}}, H_{t_{2}}\right) \leqq\left\{\int_{t_{1}}^{t_{2}} \frac{d t}{l(t)}\right\}^{-1} .
$$

Proof. Let $H_{t}^{\prime}$ be the portion of $H_{t}$ which lies between $V_{s_{1}}$ and $V_{s_{2}}$. Let $\Gamma^{\prime}=\left\{H_{t}^{\prime}\right\}_{t_{1}<t<t_{2}}$. An elementary property of extremal length gives $\lambda\left(V_{s_{1}}, V_{s_{2}} ; H_{t_{1}}, H_{t_{2}}\right) \leqq \lambda\left(\Gamma^{\prime}\right)$. Theorem 14 of [14] provides a formula for calculating the extremal length of a one-parameter family of arcs. When applied to this case the formula gives

$$
\frac{1}{\lambda\left(\Gamma^{\prime}\right)}=\int_{t_{1}}^{t_{2}} \frac{d t}{l(t)},
$$

where $l(t)$ is given by (12.2).

Let $V_{s}^{\prime}$ be the portion of $V_{s}$ between $H_{t_{1}}$ and $H_{t_{2}}$. Let $\Gamma^{\prime \prime}=$ $\left\{V_{s}^{\prime}\right\}_{s_{1}<s<s_{2}}$. Then

$$
\lambda^{-1}\left(V_{s_{1}}, V_{s_{2}} ; H_{t_{1}}, H_{t_{2}}\right)=\lambda\left(H_{t_{1}}, H_{t_{2}} ; V_{s_{1}}, V_{s_{2}}\right) \leqq \lambda\left(\Gamma^{\prime \prime}\right) .
$$

Theorem 14 [14] applied to $\Gamma^{\prime \prime}$ gives 


$$
\frac{1}{\lambda\left(\Gamma^{\prime \prime}\right)}=\int_{s_{1}}^{s_{2}} \frac{d s}{k(s)},
$$

where $k(s)$ is given by (12.1). When (12.4) and (12.5) are combined, (12.3) is obtained.

For future reference we note the corollary

$$
\int_{s_{1}}^{s_{2}} \frac{d s}{k(s)} \leqq \lambda\left(V_{s_{1}}, V_{s_{2}} ; H_{t_{1}}, H_{t_{2}}\right) \leqq \frac{1}{\left(t_{2}-t_{1}\right)^{2}} \int_{t_{1}} l(t) d t
$$

which follows from (12.3) by applying the Schwarz inequality.

\section{Applications to domains with smooth boundaries}

In this chapter we consider several explicit choices for the grid lines $\left\{V_{s}\right\},\left\{H_{t}\right\}$. In each case they can be parametrized by a differentiable mapping $\boldsymbol{c}(s, t)$ as in (11.1)-(11.3). Theorem 7 provides a tool for checking Conditions I and II. In this way Theorems 1 and 2 may be applied to yield asymptotic estimates for the mapping function $f$.

This method may be compared with that of [9], [13], [15], [16], [18]. In comparing different asymptotic estimates, no questions of sharpness can arise because the error terms have always been estimated merely as $o(1)$. The only criterion for comparison is the nature of the hypotheses under which the asymptotic expansion is valid. It will be seen that the methods of this chapter often lead to hypotheses that are less restrictive than those previously known.

13. Strip domains bounded by smooth graphs. In this section we consider regions $R$ which are bounded by the graphs of two continuously differentiable functions $\varphi_{+}>\varphi_{-}$:

$$
R=\left\{w=u+i v \mid-\infty<u<+\infty, \varphi_{-}(u)<v<\varphi_{+}(u)\right\} .
$$

Let $\zeta$ be the prime end of $R$ determined by $\lim _{\operatorname{Re} w \rightarrow+\infty} w$. Thus $f$ is a one-to-one conformal map of $R$ onto

$$
S=\{z=x+i y \mid-\infty<x<+\infty, 0<y<1\}
$$

which satisfies $\operatorname{Re} f(w) \rightarrow+\infty$ as $\operatorname{Re} w \rightarrow+\infty$.

Choose $\left\{V_{s}\right\}$ and $\left\{H_{t}\right\}$ as follows. $V_{s}$ is the Ahlfors cross cut $\left\{w=u+i v \mid u=s, \varphi_{-}(s) \leqq v \leqq \varphi_{+}(s)\right\} . H_{t}$ is the arc

$$
\left\{w=u+i v \mid v=t \varphi_{+}(u)+(1-t) \varphi_{-}(u)\right\} .
$$


Here $0 \leqq t \leqq 1$ and $s$ is any sufficiently large real number, say $s \geqq s_{0}$. These choices can be put in the form (11.1)-(11.2) by defining

$$
c(s, t)=(u, v)=\left(s, t \varphi_{+}(s)+(1-t) \varphi_{-}(s)\right) .
$$

The corresponding quantities of Theorem 7 are:

$$
\begin{aligned}
J(s, t) & =\varphi_{+}(s)-\varphi_{-}(s), \\
k(s) & =\int_{t_{1}}^{t_{2}} \frac{\left(\varphi_{+}-\varphi_{-}\right)^{2}}{\varphi_{+}-\varphi_{-}} d t=\left(t_{2}-t_{1}\right)\left(\varphi_{+}(s)-\varphi_{-}(s)\right), \\
l(t) & =\int_{s_{1}}^{s_{2}} \frac{1+\left(t \varphi_{+}^{\prime}+(1-t) \varphi_{-}^{\prime}\right)^{2}}{\varphi_{+}-\varphi_{-}} d s .
\end{aligned}
$$

Define $\vartheta(s)=\varphi_{+}(s)-\varphi_{-}(s)$. The left-hand term of $(12.6)$ is

$$
\left(t_{2}-t_{1}\right)^{-1} \int_{s_{1}}^{s_{2}} \vartheta^{-1}(s) d s
$$

The right-hand term is

$$
\begin{aligned}
& \frac{1}{\left(t_{2}-t_{1}\right)^{2}} \int_{t_{1}}^{t_{2}} \int_{s_{1}}^{s_{2}} \frac{d s d t}{\vartheta(s)}+\frac{1}{\left(t_{2}-t_{1}\right)^{2}} \int_{t_{1}}^{t_{2}} \int_{s_{1}}^{s_{2}} \frac{\left(t \varphi_{+}^{\prime}+(1-t) \varphi_{-}^{\prime}\right)^{2}}{\vartheta(s)} d s d t \\
& =\frac{1}{t_{2}-t_{1}} \int_{s_{1}}^{s_{2}} \frac{d s}{\vartheta(s)}+\frac{e\left(s_{1}, s_{2}\right)}{t_{2}-t_{1}},
\end{aligned}
$$

where

$$
0 \leqq e\left(s_{1}, s_{2}\right)<\int_{s_{1}}^{s_{2}} \frac{\varphi_{+}^{\prime 2}+\varphi_{-}^{\prime 2}}{\vartheta(s)} d s
$$

Therefore (12.6) can be written, in the present case, as

$$
\frac{1}{t_{2}-t_{1}} \int_{s_{1}}^{s_{2}} \frac{d s}{\vartheta(s)} \leqq \lambda\left(V_{s_{1}}, V_{s_{2}} ; H_{t_{1}}, H_{t_{2}}\right) \leqq \frac{1}{t_{2}-t_{1}} \int_{s_{1}}^{s_{2}} \frac{d s}{\vartheta(s)}+\frac{e\left(s_{1}, s_{2}\right)}{t_{2}-t_{1}}
$$

It follows easily from (13.4) that if

$$
e\left(s_{1}, s_{2}\right) \rightarrow 0 \quad \text { as } s_{1} \rightarrow+\infty \quad\left(s_{1}<s_{2}\right)
$$

then Conditions I and II of Chapter 1 hold. Indeed, from (13.4) we obtain, for $s_{0}<s_{1}<s<s_{2}$, 


$$
\begin{aligned}
& \lambda\left(V_{s_{1}}, V_{s_{2}} ; H_{0}, H_{1}\right)-\lambda\left(V_{s_{1}}, V_{s} ; H_{0}, H_{1}\right)-\lambda\left(V_{s}, V_{s_{2}} ; H_{0}, H_{1}\right) \\
& \leqq e\left(s_{1}, s_{2}\right)
\end{aligned}
$$

which verifies Condition I. Next consider Condition II. Define $d(s)$ by the the condition $\lambda\left(V_{s}, V_{d(s)} ; H_{0}, H_{1}\right) \equiv 1$; this is possible because $\lambda\left(V_{s}, V_{s^{\prime}} ; H_{0}, H_{1}\right)$ is a continuous function of $s^{\prime}$ (see $\left.\S 8\right)$. The lefthand inequality in (13.4) yields $\int_{s_{1}}^{s_{2}} d s / \vartheta(s) \leqq 1$, and then the righthand inequality gives

$$
\lambda\left(H_{t_{1}}, H_{t_{2}} ; V_{s}, V_{d(s)}\right) \geqq \frac{t_{2}-t_{1}}{\int_{s}^{d(s)} \frac{d s}{\vartheta(s)}+e(s, d(s))} \geqq \frac{t_{2}-t_{1}}{1+e(s, d(s))} .
$$

Therefore

$$
\lambda\left(H_{t_{1}}, H_{t_{2}} ; V_{s}, V_{d(s)}\right)-\left(t_{2}-t_{1}\right) \geqq-\frac{e(s, d(s))}{1+e(s, d(s))}
$$

which verifies Condition II; note that the parameters $a$ and $M$ of Condition II are independent of $t, a \equiv M \equiv 1$.

$\mathrm{R}$ e mark. In subsequent sections we obtain inequalities similar to (13.4). The reasoning in the preceding paragraph will be referred to in order to conclude that Condition $I$ and Condition II (uniform in $t$ ) are satisfied provided the error terms are $o(1)$ as in (13.5).

Assume (13.5) holds. Then Theorems 1 and 2 and the Remark to Theorem 2 may be applied. Theorem 1 yields

$$
\begin{aligned}
\operatorname{Re} f(u+i v) & =\lambda\left(V_{s_{0}}, V_{u} ; H_{0}, H_{1}\right)+C+o(1) \\
& =\int_{s_{0}}^{u} \frac{d s}{\varphi_{+}(s)-\varphi_{-}(s)}+C+o(1) .
\end{aligned}
$$

Theorem 2 and the Remark following it yield

$$
\begin{aligned}
\operatorname{Im} f(u+i v) & =t+o(1) & \left(u+i v \in H_{t}\right) \\
& =\frac{v-\varphi_{-}(u)}{\varphi_{+}(u)-\varphi_{-}(u)}+o(1) & (u+i v \in R) .
\end{aligned}
$$

We have proved:

Theorem 8. Let $R=\left\{w=u+i v \mid \phi_{-}(u)<v<\phi_{+}(u)\right\}$ and suppose

$$
\int_{s_{0}}^{\infty} \frac{\varphi_{+}^{\prime 2}(s)+\varphi_{-}^{\prime 2}(s)}{\varphi_{+}(s)-\varphi_{-}(s)} d s<\infty
$$


Let $f$ be a one-to-one conformal map of $R$ onto a parallel strip region $S=\{z \mid 0<\operatorname{Im} z<1\}$ such that $\operatorname{Re} f(w) \rightarrow+\infty$ as $\operatorname{Re} w \rightarrow+\infty$. Then

$$
f(u+i v)=\int_{s_{0}}^{u} \frac{d s}{\varphi_{+}(s)-\varphi_{-}(s)}+C+\frac{v-\varphi_{-}(u)}{\varphi_{+}(u)-\varphi_{-}(u)} i+E(u)
$$

where $C$ is a real constant and $\lim _{u \rightarrow+\infty} E(u)=0$.

Theorem 8 represents a strengthening of a result in [18], p. 296 and p. 323. In order to obtain (13.7) it is assumed there, in addition to (13.6), that $R$ is an $L$-strip of boundary inclination 0 which, in particular, implies that $\varphi_{ \pm}^{\prime}(s) \rightarrow 0$ as $s \rightarrow+\infty$. Theorem 8 shows that this additional condition is not needed.

14. Domains and surfaces generated by a smooth curve. In this section we consider regions $R$ which can be swept out by a line segment of varying length moving along, and normal to, a given curve.

Let $s \mapsto W(s) \in C$ be an infinite arc parametrized by arc length $s,-\infty<s<+\infty$. Let $N(s)$ be the left-hand unit normal vector. Let $\Delta(s)$ be a positive function; it will be the width of $R$ as measured along the normal $N(s)$. Assume $W$ to be of class $C^{2}$ and $\Delta$ of class $C^{1}$. We define $R$ to be the image of the mapping

$$
\begin{aligned}
(s, \tau) \mapsto w(s, \tau)= & W(s)+\tau \Delta(s) N(s) \\
& (-\infty<s<+\infty, 0<\tau<1) .
\end{aligned}
$$

We require this mapping to have positive Jacobian determinant. If the mapping is not globally one-to-one $R$ must be considered as the multisheeted image of the mapping, a Riemann surface spread over the $w$-plane.

Let $\zeta$ be the prime end of $R$ determined by the arc $w(s, 1 / 2)$ as $s \rightarrow+\infty$.

We choose $V_{s}$ to be the image under (14.1) of the vertical segment $\{(s, \tau) \mid s$ fixed, $0 \leqq \tau \leqq 1\}$. We shall consider two possibilities for choosing the family $\left\{H_{t}\right\}_{0 \leqq t \leqq 1}$. The simplest choice would be to define $H_{t}$ as the arc $s \mapsto w(s, t), s_{0} \leqq s<+\infty$. In the notation of (11.1)-(11.2) this amounts to defining $\boldsymbol{c}(s, t)$ to be $w(s, t)$.

Let $\boldsymbol{T}=\boldsymbol{W}^{\prime}$ be the unit tangent vector. The curvature $\varkappa$ satisfies $N^{\prime}=\varkappa T$. The quantities $J, k(s), l(t)$ of Theorem 7 can be calculated as follows:

$$
\begin{aligned}
c(s, t) & =W(s)+t \Delta(s) N(s), \\
\frac{\partial}{\partial s} c(s, t) & =W^{\prime}+t \Delta^{\prime} N-\varkappa t \Delta T=(1-t \varkappa \Delta) T+t \Delta^{\prime} N,
\end{aligned}
$$




$$
\begin{aligned}
\left|\frac{\partial}{\partial s} c(s, t)\right|^{2} & =(1-t \varkappa \Delta)^{2}+\left(t \Delta^{\prime}\right)^{2}, \frac{\partial}{\partial t} c(s, t)=\Delta N, \\
J(s, t) & =\left|\frac{\partial}{\partial s} c \times \frac{\partial}{\partial t} c\right|=(1-t \varkappa \Delta) \Delta, \\
k(s) & =\int_{t_{1}}^{t_{3}} \frac{\Delta d t}{1-t \varkappa \Delta}=\left.\frac{1}{-\varkappa} \log (1-t \varkappa \Delta)\right|_{t=t_{1}} ^{t_{3}}, \\
l(t) & =\int_{s_{1}}^{s_{2}} \frac{(1-t \varkappa \Delta)^{2}+t^{2} \Delta^{\prime 2}}{(1-t \varkappa \Delta) \Delta} d s .
\end{aligned}
$$

We have assumed $J>0$. We now make the stronger assumption $J \geqq \delta>0$ for some constant $\delta$. Thus

$$
(1-\varkappa \Delta) \Delta \geqq \delta>0 \text {. }
$$

In addition, assume

$$
\int_{0}^{\infty}|x| d s \text { and } \int_{0}^{\infty} \Delta^{\prime 2} d s \text { converge. }
$$

Then we obtain

$$
\frac{1}{\left(t_{2}-t_{1}\right)^{2}} \int_{t_{1}}^{t_{3}} l(t) d t \leqq \frac{1}{t_{2}-t_{1}} \int_{s_{1}}^{s_{2}} \frac{d s}{\Delta}+\frac{e\left(s_{1}, s_{2}\right)}{t_{2}-t_{1}}
$$

where $e\left(s_{1}, s_{2}\right) \rightarrow 0$ as $s_{2}>s_{1} \rightarrow+\infty$. Since

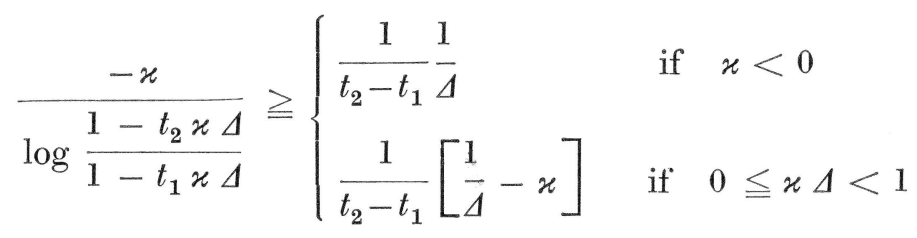

we also have

$$
\int_{s_{1}}^{s_{2}} \frac{d s}{k(s)} \geqq \frac{1}{t_{2}-t_{1}} \int_{s_{1}}^{s_{2}} \frac{d s}{\Delta}+\frac{\tilde{e}\left(s_{1}, s_{2}\right)}{t_{2}-t_{1}}
$$

where $\tilde{e}\left(s_{1}, s_{2}\right) \rightarrow 0$ as $s_{2}>s_{1} \rightarrow+\infty$. From $(14.4)-(14.5)$ we see that the present form of $(12.6)$ is 


$$
\begin{aligned}
\frac{1}{t_{2}-t_{1}} \int_{s_{1}}^{s_{2}} \frac{d s}{\Delta}+\frac{\tilde{e}\left(s_{1}, s_{2}\right)}{t_{2}-t_{1}} & \leqq \lambda\left(V_{s_{1}}, V_{s_{2}} ; H_{t_{1}}, H_{t_{2}}\right) \\
& \leqq \frac{1}{t_{2}-t_{1}} \int_{s_{1}}^{s_{2}} \frac{d s}{\Delta}+\frac{e\left(s_{1}, s_{2}\right)}{t_{2}-t_{1}}
\end{aligned}
$$

As in Section 13 (see the Remark preceding Theorem 8), this inequality leads to an asymptotic estimate for $f$. The result is:

The or e m 9. Let $R$ be the image of the mapping (14.1). If the hypotheses (14.2) - (14.3) are satisfied then

$$
f(w(s, \tau))=\int_{s_{0}}^{s} \frac{d s}{\Delta(s)}+C+i \tau+E(s)
$$

where $C$ is a real constant and $\lim _{s \rightarrow+\infty} E(s)=0$.

In the above theorem the term $\int_{s_{0}}^{s} \Delta^{-1}(s) d s$ may be replaced with $\int_{s_{0}}^{s}-[\varkappa / \log (1-x \Delta)] d s$. Thus this asymptotic expression for $\operatorname{Re} f$ is the same as that in Theorem 10 below.

Now we consider a different choice for the family $\left\{H_{t}\right\}$. Recall that $H_{t}$ should asymptotically approach the level line $\{\operatorname{Im} f=t\}$ in $R$. By way of motivation, consider the approximate behavior of $\operatorname{Im} f$ along a fixed $V_{s}$. To be definite assume $x(s)<0$. Approximate the boundary of $R$ at $W(s)$ by the osculating circle. If its radius is now increased by $\Delta(s)$ the resulting circle might be an approximation to the boundary of $R$ at $W(s)+\Delta(s) N(s)$, at least if $\Delta$ is nearly constant. Since $V_{s}$ is a radius of the annular region between these circles, we might approximate $\operatorname{Im} f$ on $V_{s}$ by the values of the harmonic measure of the annulus there. Thus at the point $w(s, \tau)$ the value $t$ of $\operatorname{Im} f$ would be approximately

$$
t=\frac{\log (1-\tau \varkappa \Delta)}{\log (1-x \Delta)}, \quad \text { so } \quad \tau=\frac{1-(1-x \Delta)^{t}}{x \Delta} .
$$

For these reasons we now choose $H_{t}$ to be the arc

$$
s \mapsto w\left(s,\left[1-(1-x \Delta)^{t}\right] / \varkappa \Delta\right) .
$$

In the notation of $(11.1)-(11.2)$ this choice for $\left\{V_{s}\right\}$ and $\left\{H_{t}\right\}$ amounts to defining $c(s, t)$ by

$$
c(s, t)=W(s)+r(s, t) N(s) \quad(-\infty<s<+\infty, 0 \leqq t \leqq 1)
$$

where

$$
r(s, t)=\frac{1}{x(s)}\left[1-(1-x(s) \Delta(s))^{t}\right]
$$


Let $T=W^{\prime}$ be the unit tangent vector. The curvature $x$ satisfies $N^{\prime}=-\varkappa T$. We now assume $x \in C^{1}$. The quantities $J, k, l$ of Theorem 7 can be calculated as follows:

$$
\begin{aligned}
\frac{\partial}{\partial s} c & =W^{\prime}+r_{s} N+r N^{\prime}=(1-r x) T+r_{s} N, \\
\left|\frac{\partial}{\partial s} c\right|^{2} & =(1-r \varkappa)^{2}+r_{s}^{2}, \\
\frac{\partial}{\partial t} c & =r_{t} N, \quad\left|\frac{\partial}{\partial t} c\right|^{2}=r_{t}^{2}, \\
r_{s}= & (1-\varkappa \Delta)^{t-1}\left\{t \Delta^{\prime}+\frac{\varkappa^{\prime}}{\varkappa^{2}}\left[1-(1-\varkappa \Delta)^{1-t}-(1-t) \varkappa \Delta\right]\right\}, \\
r_{t}= & -\frac{1}{\varkappa}(1-\varkappa \Delta)^{t} \log (1-\varkappa \Delta), \\
J(s, t)= & \left|\frac{\partial}{\partial s} c \times \frac{\partial}{\partial t} c\right|=\left|\left[(1-r \varkappa) T+r_{s} N\right] \times r_{t} N\right| \\
= & \left|(1-r \varkappa) r_{t} T \times N+r_{s} r_{t} N \times N\right| \\
= & \left(1-r \varkappa r_{t}=-\frac{1}{\varkappa}(1-\varkappa \Delta)^{2 t} \log (1-\varkappa \Delta),\right. \\
l(t)= & \int_{s_{1}} \frac{-\varkappa d s}{\log (1-\varkappa \Delta)}+\int_{s_{1}} \frac{s_{2}}{(1-\varkappa \Delta)^{t} r_{t}} \cdot \\
k(s)= & \left(t_{2}-t_{1} \frac{\log (1-\varkappa \Delta)}{-\varkappa},\right.
\end{aligned}
$$

Note that $J(s, t)$ will be positive if and only if $1-\varkappa \Delta>0$. This inequality holds because the Jacobian determinant of $w(s, \tau)$ from $(14.1)$ is $(1-\varkappa \Delta) \Delta$, and this was required to be positive in the definition of $R$.

Theorem 7 holds for unramified multisheeted coverings of plane regions as well as for plane regions. We apply this theorem, or rather its corollary (12.6), and obtain

$$
\begin{aligned}
\frac{1}{t_{2}-t_{1}} \int_{s_{1}}^{s_{2}} \frac{-\varkappa d s}{\log (1-\varkappa \Delta)} \leqq \lambda\left(V_{s_{1}}, V_{s_{2}} ; H_{t_{1}}, H_{t_{2}}\right) \\
\\
\qquad \frac{1}{t_{2}-t_{1}} \int_{s_{1}}^{s_{2}} \frac{-\varkappa d s}{\log (1-\varkappa \Delta)}+\frac{e\left(s_{1}, s_{2}, t_{1}, t_{2}\right)}{t_{2}-t_{1}}
\end{aligned}
$$


where

$$
e\left(s_{1}, s_{2}, t_{1}, t_{2}\right)-\frac{1}{t_{2}-t_{1}} \int_{t_{1}}^{t_{2}} \int_{s_{1}}^{s_{2}} \frac{r_{s}^{2} d s d t}{(1-\varkappa \Delta)^{t} r_{t}} .
$$

The integrand in (14.11) is

$$
\begin{gathered}
0 \leqq \frac{r_{s}^{2}}{(1-x \Delta)^{t} r_{t}}=\frac{\left\{t \Delta^{\prime}+\frac{x^{\prime}}{x^{2}}\left[1-(1-x \Delta)^{1-t}-(1-t) x \Delta\right]\right\}^{2}}{\Delta(1-x \Delta)^{2} \frac{\log (1-x \Delta)}{-x \Delta}} \\
\leqq \frac{2 \Delta^{\prime 2}}{\Delta} \frac{1}{(1-x \Delta)^{2} \frac{\log (1-x \Delta)}{-x \Delta}}+2 x^{\prime 2} \Delta^{3} \frac{\left[\frac{1-(1-x \Delta)^{1-t}-(1-t) x \Delta}{(x \Delta)^{2}(1-x \Delta)}\right]^{2}}{\frac{\log (1-x \Delta)}{-x \Delta}} .
\end{gathered}
$$

Assume now that $\lim \inf _{s \rightarrow+\infty}(1-\varkappa(s) \Delta(s))>0$. Then the last term above is $\left(\Delta^{\prime 2} / \Delta\right) O(1)+\varkappa^{\prime 2} \Delta^{3} O(1)$. Thus $e\left(s_{1}, s_{2}, t_{1}, t_{2}\right) \rightarrow 0$ as $s_{2}>s_{1} \rightarrow+\infty$, uniformly for $0 \leqq t_{1}<t_{2} \leqq 1$, provided

$$
\int_{0}^{\infty} \frac{\Delta^{\prime 2}}{\Delta} d s<\infty, \quad \int_{0}^{\infty} \varkappa^{\prime 2} \Delta^{3} d s<\infty, \quad \liminf _{s \rightarrow+\infty}(1-\varkappa \Delta)>0 .
$$

As in Section 13, the inequalities (14.10) and the uniform behavior of $e\left(s_{1}, s_{2}, t_{1}, t_{2}\right) \rightarrow 0$ show that Theorems 1, 2 and the Remark following it can be applied (see the Remark preceding Theorem 8). Theorem 1 yields

$$
\operatorname{Re} f(w(s, \tau))=\int_{0}^{s} \frac{-\varkappa d s}{\log (1-\varkappa \Delta)}+C+o(1), \quad \text { as } s \rightarrow+\infty .
$$

Theorem 2 and the Remark following it yield

$$
\begin{array}{rlrl}
\operatorname{Im} f(w(s, \tau)) & =t+o(1) & & \left(\text { if } w(s, \tau) \in H_{t}\right) \\
& =\frac{\log (1-\tau \varkappa \Delta)}{\log (1-\varkappa \Delta)}+o(1) & (\text { all } w(s, \tau) \in R)
\end{array}
$$

(see (14.7)). We have proved:

The or em 10. Let $R$ be the image of the mapping (14.1). If the hypotheses (14.12) are satisfied then 


$$
f(w(s, \tau))=\int_{0}^{s} \frac{-\varkappa d s}{\log (1-\varkappa \Delta)}+C+i \frac{\log (1-\tau \varkappa \Delta)}{\log (1-\varkappa \Delta)}+E(s)
$$

where $C$ is a real constant and $\lim _{s \rightarrow+\infty} E(s)=0$.

When $\Delta(s) \equiv$ const. $=1$, Theorem 10 yields a result of Stročik [15], which he proved by use of the Teichmüller - Wittich - Belinsky Theorem. In this paper the strip $R$ is defined (using our notation) by the mapping $(s, \tau) \mapsto w(s, \tau)=W(s)+\tau N,-1 / 2<\tau<1 / 2$; thus $W(s)$ is the "center" curve of $R$ rather than the "lower" boundary. An elementary calculation shows that his hypotheses, in terms of our quantities, require that $\int_{0}^{\infty} \operatorname{Max}\left(x^{\prime 2},\left|x^{\prime}\right|\right) d s<\infty$, and that for some $\delta, 0<\delta<$ $1,-2(1-\delta) / \delta<\varkappa<1-\delta$. These conditions are more restrictive than ours (in particular we do not need the convergence of $\int_{0}^{\infty}\left|x^{\prime}\right| d s$ ).

Theorems 9 and 10 may also be compared with Theorem IX of [18]. Despite some similarity in the form of the asymptotic expansion of $f(w)$, it is easily seen that the result in [18] neither contains nor is contained in our present theorems.

15. Symmetric strip domains. In this section we mention a special case of the situation treated in Section 13; namely, the case that $\varphi_{-}=-\varphi_{+}$. This situation was treated in Gol'dberg - Stročik [9] by a method based on the Teichmüller - Wittich - Belinsky Theorem.

The results of [9] can also be obtained by the methods of this chapter. This is done by defining $\boldsymbol{c}(s, t)$ as follows (we write $\varphi$ for $\varphi_{+}$):

$$
c(s, t)=(P(s)+r(s) \cos \tau(s, t), r(s) \sin \tau(s, t))
$$

where

$$
\begin{aligned}
P(s) & =s-\frac{\varphi(s)}{\varphi^{\prime}(s)}, \quad r(s)=\frac{\varphi(s)}{\varphi^{\prime}(s)} \sqrt{1+\varphi^{\prime 2}(s)}, \\
\tau(s, t) & =(2 t-1) \arctan \varphi^{\prime}(s) .
\end{aligned}
$$

Geometrically, $P(s)$ is the center of a circle of radius $r(s)$ which is orthogonal to the boundary of $R$ at the points $(s, \pm \varphi(s))$. The cross cut $V_{s}$ is an arc of this circle, and $H_{t}$ divides $V_{s}$ into two arcs whose lengths have the ratio $t:(1-t)$.

We assume that $\varphi$ is twice continuously differentiable. Then the condition

$$
\liminf _{s \rightarrow+\infty} \frac{\varphi \varphi^{\prime \prime}}{\left(1+\varphi^{\prime 2}\right)\left(1+\sqrt{\left.1+\varphi^{\prime 2}\right)}\right.}>-1
$$

ensures that the mapping $(s, t) \mapsto c(s, t)$ is one-to-one and that $J>0$. 
Thus, given $w=u+i v \in R$ there exists a unique pair $(s, t)$ satisfying (15.1).

When the techniques of this chapter are applied, we obtain (we omit the details of the calculations):

$\left(t_{2}-t_{1}\right) \int_{s_{1}}^{s_{2}} \frac{d s}{k(s)} \geqq \int_{s_{1}}^{s_{2}} \frac{\varphi^{\prime} d s}{2 \varphi \arctan \varphi^{\prime}}+\frac{1}{2} \int_{s_{1}}^{s_{2}} \frac{\varphi^{\prime}}{\arctan \varphi^{\prime}} \frac{\min \left(0, \varphi^{\prime \prime}\right)}{\left(1+\varphi^{\prime 2}\right)\left(1+\sqrt{\left.1+\varphi^{\prime 2}\right)}\right.} d s$

and

$$
\begin{aligned}
& \frac{1}{t_{2}-t_{1}} \int_{t_{1}}^{t_{2}} l(t) d t \\
& \leqq \int_{s_{1}}^{s_{2}} \frac{\varphi^{\prime} d s}{2 \varphi \arctan \varphi^{\prime}}+M \int_{s_{1}}^{s_{2}}\left\{\frac{\left|\varphi^{\prime \prime}\right|}{\left(1+\varphi^{\prime 2}\right)^{3 / 2}}+\frac{\varphi\left(\varphi^{\prime \prime}\right)^{2}}{\left(1+\varphi^{\prime 2}\right)^{3}}\right\} \frac{\varphi^{\prime} d s}{\arctan \varphi^{\prime}},
\end{aligned}
$$

where $M$ is a positive constant. Thus

$$
\begin{aligned}
\frac{1}{t_{2}-t_{1}}\left\{\int_{s_{1}}^{s_{2}} \frac{\varphi^{\prime} d s}{2 \varphi \arctan \varphi^{\prime}}-\tilde{e}\left(s_{1}, s_{2}\right)\right\} & \leqq \lambda\left(V_{s_{1}}, V_{s_{2}} ; H_{t_{1}}, H_{t_{2}}\right) \\
& \leqq \frac{1}{t_{2}-t_{1}}\left\{\int_{s_{1}}^{s_{2}} \frac{\varphi^{\prime} d s}{2 \varphi \arctan \varphi^{\prime}}+e\left(s_{1}, s_{2}\right)\right\}
\end{aligned}
$$

where

$$
0 \leqq \tilde{e}\left(s_{1}, s_{2}\right)=O\left\{\int_{s_{1}}^{s_{2}} \frac{\left|\varphi^{\prime \prime}\right| d s}{1+\varphi^{\prime 2}}\right\}
$$

and

$$
0 \leqq e\left(s_{1}, s_{2}\right)=O\left\{\int_{s_{1}}^{s_{2}} \frac{\left|\varphi^{\prime \prime}\right|}{1+\varphi^{\prime 2}} d s+\int_{s_{1}}^{s_{2}} \frac{\varphi\left(\varphi^{\prime \prime}\right)^{2}}{1+\left|\varphi^{\prime}\right|^{5}} d s\right\}
$$

Following an argument similar to that in Section 13 we obtain:

Th e o r e m 11. (Gol'dberg-Stročik [9].) If in addition to (15.3), $\varphi$ satisfies the conditions

$$
\int_{s_{0}}^{\infty} \frac{\left|\varphi^{\prime \prime}\right|}{1+\varphi^{\prime 2}} d s<\infty \quad \text { and } \quad \int_{s_{0}}^{\infty} \frac{\varphi\left(\varphi^{\prime \prime}\right)^{2}}{1+\left|\varphi^{\prime}\right|^{5}} d s<\infty
$$

then 


$$
f(c(s, t))=\int_{s_{0}}^{s} \frac{\varphi^{\prime} d s}{2 \varphi \arctan \varphi^{\prime}}+i t+C+E(s)
$$

where $C$ is a real constant and $\lim _{s \rightarrow+\infty} E(s)=0$.

\section{References}

[1] AHLFORS, L. V.: Untersuchungen zur Theorie der konformen Abbildung und der ganzen Funktionen. - Acta Soc. Sci. Fenn. (Nova Ser.) A. 1:9, $1930,1-40$.

[2] - - Conformal invariants. Topics in geometric function theory. - McGrawHill Book Company, New York etc., 1973.

[3] EкE, B. G.: Remarks on Ahlfors' distortion theorem. - J. Analyse Math. 19, 1967, 97-134.

[4] - - - On the angular derivative of regular functions. - Math. Scand. 21, 1967, $122-127$.

[5] - - - On the differentiability of conformal maps at the boundary. - Nagoya Math. J. 41, 1971, 43-53.

[6] - - Comparison domains for the problem of the angular derivative. - Comment. Math. Helv. 46, 1971, 98-112.

[7] CatTegno, C., and A. Ostrowski: Représentation conforme à la frontière; domaines généraux. - Mémor. Sci. Math. 109, 1949, 1-60.

[8] -》- - - Représentation conforme à la frontière; domaines particuliers. - Mémor. Sci. Math. 110, 1949, 1-56.

[9] Gol'DberG, A. A., and T. V. STročiK [A. А. Гольдберг and T. В. Строчик]: Конформное отображение симметричных полуполос и угловых областей. Zusammenfassung: Konforme Abbildung von symmetrischen Halbstreifen und Eckengebieten. - Litovsk. Mat. Sb. 6, 1966, 227-239.

[10] JACKson, H. L.: Some remarks on angular derivatives and Julia's lemma. - Canad. Math. Bull. 9, 1966, 233-241.

[11] Jenkins, J. A., and K. OtKawa: On results of Ahlfors and Hayman. - Illinois J. Math. 15, 1971, 664-671.

[12] LEIONG - FERRAND, J.: Représentation conforme et transformations à intégrale de Dirichlet bornée. - Cahiers Scientifiques 22. Gauthier-Villars, Éditeur, Paris, 1955.

[13] OBRock, A. E.: On bounded oscillation and asymptotic expansion of conformal strip mappings. - Trans. Amer. Math. Soc. 173, 1972, 183-201.

[14] Rodin, B.: The method of extremal length. - Bull. Amer. Math. Soc. 80, 1974, 587-606.

[15] STročIK, T. V. [T. В. Строчик]: О конформном отображении полуполос постоянной ширины. - Ukrain. Mat. ̌̌. 21, 1969, 60-72. Translation: On conformal mappings of a semistrip of constant width. - Ukrainian Math. J. $21,1969,48-57$.

[16] -»- О конформном отображении одного класса полуполос. - Sibirsk. Mat. Ž. 11, 1970, 859-878. Translation: Conformal mapping of a class of semistrips. - Siberian Math. J. 11, 1970, 647-661. 
[17] TEICHMÜLLER, O.: Untersuchungen über konforme und quasikonforme Abbildung. - Deutsche Math. 3, 1938, 621-678.

[18] Warschawski, S. E.: On conformal mapping of infinite strips. - Trans. Amer. Math. Soc. 51, 1942, 280-335.

[19] -»- On the boundary behavior of conformal maps. - Nagoya Math. J. 30, 1967, $83-101$.

[20] -»- Remarks on the angular derivative. - Nagoya Math. J. 41, 1971, $19-32$.

(Added in proof.) The work of J.A. Jenkins and K. Oikawa referred to in footnote 1, p. 484, is Conformality and semiconformality at the boundary to appear in J. Reine Angew. Math.

University of California, San Diego

Department of Mathematics

La Jolla, California 92093

USA

Received 27 February 1975 